\title{
SERGE COHEN
}

ANNE ESTRADE

\section{Régularisation d'équations de Stratonovitch} à sauts entre variétés

\author{
Annales de la faculté des sciences de Toulouse $6^{e}$ série, tome 7, $\mathrm{n}^{\circ} 2$ \\ (1998), p. 233-265 \\ <http://www.numdam.org/item?id=AFST_1998_6_7_2_233_0>
}

(C) Université Paul Sabatier, 1998, tous droits réservés.

L'accès aux archives de la revue «Annales de la faculté des sciences de Toulouse » (http://picard.ups-tlse.fr/ annales/) implique l'accord avec les conditions générales d'utilisation (http://www.numdam.org/conditions). Toute utilisation commerciale ou impression systématique est constitutive d'une infraction pénale. Toute copie ou impression de ce fichier doit contenir la présente mention de copyright.

\section{NumDam}

Article numérisé dans le cadre du programme

Numérisation de documents anciens mathématiques

http://www.numdam.org/ 


\title{
Régularisation d'équations de Stratonovitch à sauts entre variétés $\left.{ }^{*}\right)$
}

\author{
${\text { Serge } \operatorname{Cohen}^{(1)} \text { et Anne Estrade }}^{(2)}$
}

RÉSUME. - Nous présentons ici un procédé géométrique pour régulariser des semi-martingales càdlàg. On en déduit que les solutions de certaines équations différentielles stochastiques sont des limites de solutions d'équations ordinaires, où le bruit stochastique a d'abord été régularisé.

Notre démarche est la suivante. Pour lisser une semi-martingale à valeurs dans une variété, nous fixons une trajectoire et nous approchons sa position à l'instant $t$ par une "moyenne" des points de la trajectoire sur un petit intervalle de temps avant $t$. On construit cette moyenne en généralisant à la variété la notion de barycentre des espaces vectoriels et on obtient une suite de processus continus et à variation finie qui convergent vers la semi-martingale initiale. La convergence est suffisamment forte pour entraîner celle des solutions d'équations différentielles ordinaires dirigées par ces processus, et la limite s'interprète naturellement comme la solution d'une équation différentielle stochastique entre variétés. Ce travail entre dans le cadre des approximations Wong-Zakaï d'équations de Stratonovitch, et fournit une extension géométrique du calcul de Stratonovitch à sauts proposé par Kurtz, Pardoux et Protter.

Le caractère intrinsèque de notre méthode permet d'obtenir des résultats de convergence en l'absence de structure linéaire, même quand la source présente des discontinuités.

ABstract. - We investigate a way to regularize a general manifold valued semimartingale, in order to approximate the solution of some stochastic differential equation. A regularization of the stochastic driving noise is used. The regularization procedure is the following: a sample path of the càdlàg manifold valued semimartingale is fixed, and the

(*) Reçu le 25 mars 1996, accepté le 13 mai 1997

(1) C.E.R.M.I.C.S. E.N.P.C., 6 et 8 avenue Blaise-Pascal, Cité Descartes - Champssur-Marne, F-77455 Marne-La-Vallée Cedex 2

Département de Mathématiques, Université de Versailles, St-Quentin-en-Yvelines, 45 avenue des États-Unis, F-78035 Versailles (France)

e-mail : cohen@cermat.math-uvsq.fr

(2) M.A.P.M.O. - U.M.R. 6628, U.F.R. Sciences, Université d'Orléans, B.P. 6759,

F-45067 Orléans Cedex 2 (France)

E-mail : estrade@labomath.univ-orleans.fr 
semimartingale position at time $t$ is approximated by a "mean value" of the sample path just before time $t$. The usual definition of vector valued barycenter has to be extended to construct this intrinsic "mean value", and the resulting regularized processes, which are continuous with finite variation, produce an estimate of the original semimartingale. The solutions of the ordinary differential equations driven by those finite variation processes are proved to converge to the so-called solutions of stochastic differential equations between manifolds. This "Wong-Zakai" type approximation of a Stratonovich differential equation is a natural geometrical extension of a paper by Kurtz, Pardoux and Protter. At last we would like to emphasize that the geometric point of view allows us to deal with a discontinuous driving process even if the regularization is not linear.

AMS Classification : Primary 58G32, 60J75; secondary 58F30, 60H20.

MOTS-CLEs : Géométrie différeniielle stochastique, Processus de sauts, Calcul de Stratonovitch.

\section{Introduction}

Nous nous intéressons à la régularisation de semi-martingales discontinues à valeurs dans une variété, ainsi qu'à ses conséquences sur les solutions d'EDS entre variétés dirigées par ces semi-martingales. Le point de départ de cet article est le travail de Kurtz, Pardoux et Protter consacré aux EDS de Stratonovitch dirigées par des semi-martingales réelles générales [8]. Entre autres propriétés, ils montrent un résultat d'approximation de type "WongZakai" : une semi-martingale discontinue est approchée par des processus à variation finie et les solutions des équations différentielles ordinaires dirigées par ces processus réguliers convergent vers la solution de l'EDS de Stratonovitch initiale. Depuis l'article initial [13], beaucoup d'autres approximations ont été proposées, y compris dans le cas où la semi-martingale présente des sauts. On peut ainsi citer un article de Protter [11] dans lequel des semimartingales vectorielles discontinues sont approchées de façon polygonale. Cependant on montre ici qu'une structure linéaire n'est pas indispensable pour la régularisation : notre semi-martingale est à valeurs dans une variété et le procédé est intrinsèque. Ceci a plusieurs conséquences. Tout d'abord, dans le cadre de la géométrie différentielle stochastique, la régularisation de semi-martingales dans une variété présente un intérêt propre; aussi bien du point de vue de la simulation que de la modélisation, il est utile de pouvoir 
considérer les processus discontinus comme des limites de processus continus à variation finie. Une telle approche ramène en effet les EDS avec sauts entre variétés au cadre classique du calcul de Stratonovitch : les processus continus. Ensuite, cette présentation géométrique montre que, même pour des semi-martingales vectorielles, on dispose de plusieurs procédés de régularisation en dehors de celui proposé dans [8]. Enfin la flexibilité de notre technique provient de l'utilisation d'arguments de compacités généraux, et permet de surmonter les problèmes posés par la non-linéarité et les discontinuités.

Décrivons plus en détail notre méthode. Le but premier est donc d'approcher une semi-martingale càdlàg à valeurs dans une variété, par une famille de processus $\left(X^{h}\right)_{h}$ définis intrinsèquement. On demande que les processus $X^{h}$ soient suffisamment réguliers, à savoir continus à variation finie, et que la convergence des $X^{h}$ vers $X$ soit suffisamment forte pour entraîner celle des solutions des EDS associées. Le procédé repose sur une idée classique : remplacer la valeur de $X$ à l'instant $t$ par la "valeur moyenne" de $X$ sur l'intervalle $[t-h, t]$. Il s'agit d'abord de donner un sens intrinsèque à la valeur moyenne sur une variété. Les barycentres introduits par Picard [10], tout comme les interpolateurs utilisés par Cohen [3], fournissent un outil adapté. Nous obtenons ainsi une famille $\left(X^{h}\right)_{h}$ de processus continus à variation finie qui converge presque sûrement vers $X_{-}$. Pour obtenir la convergence des solutions des EDS associées, il est nécessaire d'effectuer un changement de temps sur les processus $X^{h}$ suivant l'idée de Kurtz dans [7]; on ouvre ainsi des intervalles de temps fictifs aux instants de sauts de $X$. Ensuite nous appliquons cette technique aux EDS à sauts entre variétés, telles qu'elles sont décrites dans [8]. Étant données deux variétés $M$ et $N$, une famille suffisamment régulière $\{e(x, y) \mid x \in M, y \in N\}$, où $e(x, y)$ est une application linéaire de $T_{x} M$ dans $T_{y} N$, et une semi-martingale càdlàg $X$ à valeurs dans $M$, nous regardons les équations différentielles ordinaires dirigées par les régularisées $X^{h}$ et associées à l'opérateur $e$ :

$$
\mathrm{d} Y^{h}=e\left(X^{h}, Y^{h}\right) \mathrm{d} X^{h} .
$$

Comme dans le cas réel, nous montrons que la famille $\left(Y^{h}\right)_{h}$ converge, à un changement de temps près, vers la solution de l'EDS de Stratonovitch, dirigée par $X$ et associée à l'opérateur $e$.

Notre travail se découpe en trois parties. La première est consacrée à un rappel rapide des résultats de [8]. La régularisation d'une semi-martingale discontinue $X$ sur une variété fait l'objet du second paragraphe. Dans la 
troisième partie, nous étudions l'effet de la régularisation sur les EDS. Le théorème principal est énoncé au paragraphe 3.2, accompagné de rappels sur les EDS à sauts entre variétés, et la démonstration est présentée au paragraphe 3.3.

\section{Approximation dans le cas réel}

Nous présentons ici les résultats d'approximation qu'ont obtenus Kurtz, Pardoux et Protter dans [8], dans le but de motiver l'introduction des différents objets mathématiques et de fixer les notations.

Soit $(\Omega, \mathcal{F}, \mathbf{P})$ un espace de probabilité, muni d'une filtration $\left(\mathcal{F}_{t}\right)_{t}$ satisfaisant aux conditions habituelles. Soit $X$ une semi-martingale définie sur cet espace, càdlàg, à valeurs réelles. On note $[X, X]$ le processus croissant associé qui se décompose en une partie continue $[X, X]^{c}$ et une partie totalement discontinue $[X, X]^{d}$. Pour toute fonction $f$ de classe $C^{1}$ de $\mathbb{R}$ dans $\mathbb{R}$, telle que $f$ et $f^{\prime}$ soient bornées et lipschitziennes, et pour toute variable aléatoire $Y_{0} \mathcal{F}_{0}$-mesurable, on introduit l'équation différentielle stochastique suivante :

$$
\begin{aligned}
Y_{t}= & Y_{0}+\int_{0}^{t} f\left(Y_{s^{-}}\right) \mathrm{d} X_{s}+\frac{1}{2} \int_{0}^{t} f^{\prime} f\left(Y_{s}\right) \mathrm{d}[X, X]_{s}^{c} \\
& +\sum_{0<s \leq t}\left(\varphi\left(f, \Delta X_{s}, Y_{s^{-}}\right)-Y_{s^{-}}-f\left(Y_{s^{-}}\right) \Delta X_{s}\right)
\end{aligned}
$$

où, pour $g \in \mathcal{C}^{1}(\mathbb{R}, \mathbb{R})$ et $x, y \in \mathbb{R}, \varphi(g, x, y)$ désigne la valeur à l'instant 1 de la solution de l'équation différentielle :

$$
\left\{\begin{array}{l}
y^{\prime}(u)=g(y(u)) x \\
y(0)=y
\end{array}\right.
$$

Il faut insister sur le fait que l'équation (1) est une équation de Stratonovitch, ce qui est naturel pour une limite d'approximations de type "WongZakai". Ainsi les deux premiers termes intégraux de (1) sont classiques pour une équation de Stratonovitch quand la semi-martingale $X$ est continue. Le troisième terme précise le comportement de la solution aux instants de sauts de $X$. Il est établi (théorème 3.2 de [8]) que pour toute variable aléatoire $Y_{0}$, l'équation (1) admet une unique solution, qui est une semi-martingale. 
Ensuite, la semi-martingale directrice de l'EDS (1) est approchée grâce à un procédé régularisant. Pour tout $h>0$, on considère la semi-martingale $X^{h}$ définie par

$$
X_{t}^{h}=\frac{1}{h} \int_{t-h}^{t} X_{s} \mathrm{~d} s
$$

Le processus $X^{h}$ est continu, à variation finie sur tout compact et, pour tout $t>0,\left(X_{t}^{h}\right)_{h}$ tend presque sûrement vers $X_{t^{-}}$quand $h$ tend vers $0^{+}$. Comme cette formule impose que l'on fixe une définition pour la valeur de $X$ en des temps négatifs $(t<0)$, on supposera dans la suite de l'article que tous les processus a priori définis pour $t \geq 0$, sont constants et égaux à leurs valeurs en 0 , pour $t<0$. Ainsi prolongés les processus sont continus en $t=0$.

On s'intéresse ensuite à la famille des processus $Y^{h}$, solution pour chaque $h>0$, de l'équation différentielle ordinaire

$$
Y_{t}^{h}=Y_{0}+\int_{0}^{t} f\left(Y_{s}^{h}\right) \mathrm{d} X_{s}^{h} .
$$

Les processus $Y^{h}$ convergent, "à un changement de temps près", vers la solution de l'équation (1). Le changement de temps est donné, pour tout $h>0$, par

$$
\begin{aligned}
\gamma_{h}(t) & =\frac{1}{h} \int_{t-h}^{t}\left([X, X]_{s}^{d}+s\right) \mathrm{d} s \\
\gamma(t) & =[X, X]_{t}^{d}+t,
\end{aligned}
$$

et le type de convergence est précisé dans le théorème suivant ([8, théorème 6.5]).

THÉORÈME 1.1. - La famille $\left(Y^{h} \circ \gamma_{h}^{-1}\right)_{h}$ converge uniformément sur tout compact en probabilité vers un processus adapté continu $\widehat{Y}$ vérifiant $Y=\widehat{Y} \circ \gamma$.

Comme conséquence de ce théorème, on obtient la convergence en probabilité de $\left(Y_{t}^{h}\right)_{h}$ vers $Y_{t}$ en dehors des instants de discontinuité de $\gamma$ (qui sont aussi ceux de $X$ ).

\section{Régularisation de semi-martingale sur une variété}

Dans cette section, nous considérons une semi-martingale $X$ càdlàg à valeurs dans une variété. Comme dans le cas réel, il s'agit tout d'abord 
de construire une famille de semi-martingales $X^{h}$ plus régulières que $X$, qui approche $X$. Kurtz, Pardoux et Protter ont choisi pour $X_{t}^{h}$ la valeur moyenne de $X$ sur $[t-h, t]$ (voir (2)). Nous avons opté pour un procédé de régularisation analogue, réalisable dans le cadre de processus à valeurs dans une variété, en remplaçant la moyenne par une projection sur $M$ de l'ensemble $\mathcal{P}$ des probabilités sur la variété $M$. Pour conserver la propriété de régularisation, la projection sera supposée localement lipschitzienne. On reconnait ainsi une extension de la notion de barycentre, introduite par Picard dans [10] pour étudier des martingales sur une variété. Bien que cette projection soit plus proche des interpolateurs de [3] et du calcul de Stratonovitch, que des connecteurs et du calcul d'Itô, nous conserverons le terme de barycentre.

Par ailleurs, précisons les hypothèses concernant la géométrie que nous ferons dans la suite. Les variétés seront toujours avec ou sans bord, connexes, de classe $C^{3}$ au moins. Sur chaque variété $M$, on choisira toujours une distance $d_{M}$ provenant d'un plongement dans $\mathbb{R}^{m}$ ou de cartes locales. Ces distances, que nous appellerons bonnes distances, dépendent évidemment $\mathrm{du}$ choix du plongement ou des cartes locales; en revanche, elles sont toutes localement équivalentes.

Enfin, une bonne distance $d_{M}$ sur $M$ étant fixée, nous définissons la variance d'une variable aléatoire dans $M$.

DÉFINITION 2.1.- Si $X$ est une variable aléatoire à support compact dans $M$, on appelle variance de $X$ la quantité

$$
\operatorname{var} X=\inf _{x \in M} \mathbf{E}\left(d_{M}(x, X)^{2}\right) .
$$

Cette définition dépend de la distance $d_{M}$ choisie mais, par équivalence des bonnes distances, l'ordre de grandeur lui, est intrinsèque quand on suppose les variables à support dans un compact fixe.

On dispose maintenant du vocabulaire nécessaire pour définir un barycentre.

\subsection{Barycentre sur une variété}

Soit $\mathcal{P}$ l'espace des mesures de probabilité à support compact dans $M$.

DÉfInItIon 2.2.- Une application $\mathbf{B}$ de $\mathcal{P}$ sur $M$ sera appelée barycentre si elle vérifie les propriétés suivantes. 
a) Si $\delta_{x}$ est la masse de Dirac au point $x$ de $M$,

$$
\mathbf{B}\left(\delta_{x}\right)=x \text {. }
$$

b) Pour tout compact $K$ de $M$, il existe une constante $C_{K}$ telle que pour tout couple de variables aléatoires $\left(X_{1}, X_{2}\right)$ à valeurs dans $K$,

$$
d_{M}\left(\mathbf{B}\left(\mathbf{P} \circ X_{1}^{-1}\right), \mathbf{B}\left(\mathbf{P} \circ X_{2}^{-1}\right)\right) \leq C_{K} \mathbf{E}\left(d_{M}\left(X_{1}, X_{2}\right)\right) .
$$

c) En plongeant $M$ dans $\mathbb{R}^{m}$, pour toute variable aléatoire $X$ à valeurs dans un compact de $M$,

$$
\mathbf{E}(X)-\mathbf{B}\left(\mathbf{P} \circ X^{-1}\right)=\mathrm{O}(\operatorname{var} X)
$$

quand $\operatorname{var} X$ tend vers 0 .

Remarque 2.3. - L'équivalence des bonnes distances montre que les propriétés b) et c) ne dépendent pas du choix d'une bonne distance particulière.

Remarque 2.4. - La propriété de Lipschitz b) assure la continuité de l'application $\mathbf{B}$ sur l'espace $\mathcal{P}_{K}$ des lois ayant un support contenu dans un compact fixe $K$. Cela suffit pour régulariser une semi-martingale au moyen d'un barycentre, car quitte à arrêter les différents processus considérés par des temps de sortie de compacts, on peut supposer $M$ compact sans restreindre la généralité des résultats.

Remarque 2.5. - Si B est un barycentre et si on note $I$ l'application de $[0,1] \times M \times M$ dans $M$ définie par

$$
I(t, x, z)=\mathbf{B}\left((1-t) \delta_{x}+t \delta_{z}\right),
$$

alors la différentielle des applications partielles $I_{t, x}=I(t, x, \cdot)$ au point $z=x$ vérifie

$$
\mathrm{d}_{x}\left(I_{t, x}\right)=t \operatorname{Id}_{T_{x} M} .
$$

Preuve. - Soient $(t, x, z) \in[0,1] \times M \times M$ et $X_{t}^{x, z}$ une variable aléatoire qui prend les valeurs $x$ et $z$ avec les probabilités respectives $1-t$ et $t$. On plonge $M$ dans $\mathbb{R}^{m}$ et la condition c) de la définition 2.2 permet alors d'écrire dans $\mathbb{R}^{m}$ un développement limité de $I_{t, x}$ au voisinage de $x$ :

$$
\begin{aligned}
I_{t, x}(z) & =E\left(X_{t}^{x, z}\right)+\mathrm{O}\left(\operatorname{var}\left(X_{t}^{x, z}\right)\right. \\
& =(1-t) x+t z+\mathrm{O}\left(t d_{M}(x, z)^{2}\right) .
\end{aligned}
$$

On vérifie ainsi que $\mathrm{d}_{x}\left(I_{t, x}\right)=t \operatorname{Id}_{T_{x} M}$. 
L'application I définie par (4) est donc un interpolateur au sens de la définition 10 de [10]. Par la suite, nous utiliserons cet interpolateur pour définir un calcul de Stratonovitch intrinsèque, et pour le moment, nous le baptisons.

DÉFINITION 2.6. - Si $\mathbf{B}$ est un barycentre, nous appellerons interpolateur associé à B l'application I de $[0,1] \times M \times M$ dans $M$ donnée par (4), et nous dirons que $\mathbf{B}$ est de classe $C^{r}$ si $I$ est de classe $C^{r}$.

Nous avons étendu la notion de barycentre introduite par Picard ([10, Prop. 2.1]) en localisant l'hypothèse de Lipschitz. Cependant les restrictions topologiques évoquées dans [10], si la variété $M$ est compacte, demeurent. En effet, une variété possédant un interpolateur continu est contractible, ce qui exclut les variétés compactes sans bord.

Dans toute la suite, nous supposerons fixé un barycentre $\mathbf{B}$ de classe $C^{3}$.

\subsection{Définition et propriétés de $X^{h}$}

Nous appellerons régularisée de $X$ la suite de processus $X^{h}$ obtenue de la manière suivante.

DÉfINITION 2.7. - Soit $X$ une semi-martingale càdlàg à valeurs dans $M$. Pour tout $h>0$ et tout $t \geq 0$, nous définissons (trajectoire par trajectoire) $X_{t}^{h}$ comme étant le barycentre des $X_{s}$, où s parcourt de manière uniforme l'intervalle de temps $[t-h, t]$. Autrement dit, en notant $\mu_{t}^{h}$ la mesure de probabilité uniforme sur $[t-h, t]$, pour presque tout $\omega \in \Omega$, $\mu_{t}^{h} \circ X(\omega)^{-1}$ désigne la mesure image sur $M$ de $\mu_{t}^{h}$ par la variable aléatoire $X(\omega):[t-h, t] \longrightarrow M$, et on pose

$$
X_{t}^{h}(\omega)=\mathbf{B}\left(\mu_{t}^{h} \circ X(\omega)^{-1}\right) .
$$

Il est clair que si $X$ est à valeurs dans un espace vectoriel et que l'on choisit le barycentre naturellement associé à l'espérance d'une variable aléatoire, on trouve la régularisation de [8]. En effet si $Q$ est une probabilité à support dans un compact de $\mathbb{R}^{m}$, et si l'on pose $\mathbf{B}(Q)=\int x \mathrm{~d} Q(x)$, alors $X_{t}^{h}=(1 / h) \int_{t-h}^{t} X_{s} \mathrm{~d} s$.

Illustrons ce procédé géométrique de régularisation en choisissant des exemples de barycentres. Si $M$ est une sous-variété de $\mathbb{R}^{m}$, et $\pi$ une 
projection de l'enveloppe convexe de $M$ dans $M$, il est naturel de poser pour une probabilité $Q$ à support compact dans $M$,

$$
\mathbf{B}(Q)=\pi\left(\int_{\mathbb{R}^{m}} x \mathrm{~d} Q(x)\right) .
$$

C'est un barycente d'interpolateur associé $I(t, x, z)=\pi(t x+(1-t) z)$. On peut regarder un cas particulier de ce modèle et considérer un hémisphère de la sphère de dimension $2, M=\left\{x=\left(x_{1}, x_{2}, x_{3}\right) \in \mathbb{R}^{3} \mid x_{3}>0\right.$ et $\left.\|x\|=1\right\}$ où $\|x\|$ est la norme euclidienne de $x$ dans $\mathbb{R}^{3}$. On vérifie aisément que, si $X$ est à valeurs dans l'hémisphère, les régularisées $X^{h}$ sont données par

$$
X_{t}^{h}=\frac{\frac{1}{h} \int_{t-h}^{t} X_{s} \mathrm{~d} s}{\left\|\frac{1}{h} \int_{t-h}^{t} X_{s} \mathrm{~d} s\right\|},
$$

et sont ainsi des semi-martingales continues à valeurs dans l'hémisp ère.

Rappelons par ailleurs que si $M$ est une variété riemannienne et $\delta$ ést une distance sur $M$ issue de la métrique riemannienne, on peut construire un barycentre métrique. Pour que cet objet soit défini, il faut supposer que pour toute variable aléatoire $X$, la fonction $x \in M \longmapsto \mathbf{E}\left(\delta^{2}(X, x)\right)$ admet un unique point critique non dégénéré. Cette hypothèse est notamment vérifiée pour les variétés de Cartan-Hadamard. On appelle alors barycentre métrique de toute variable aléatoire cet unique point critique qui est un minimum. Dans cet exemple l'interpolateur $I(\cdot, x, z)$ est l'unique géodésique qui va de $x$ à $z$, et le régularisé $X_{t}^{h}$ est l'unique minimum de $\int_{t-h}^{t} \delta^{2}\left(X_{s}, x\right) \mathrm{d} s$ en tant que fonction de $x$.

Revenons au cas général. Le lemme suivant nous assure que les $X^{h}$, ainsi définis, remplissent leur rôle de régularisation.

Lemme 2.8. - Presque sûrement,

(i) pour tout $h>0$, l'application $t \mapsto X_{t}^{h}$ est continue, à variation finie sur tout compact;

(ii) pour tout $t \in \mathbb{R}_{+},\left(X_{t}^{h}\right)_{h>0}$ tend vers $X_{t^{-}}$quand $h$ tend vers 0 .

\section{Preuve}

(i) On fixe une trajectoire de $X$ et un réel $h>0$. Ainsi on pourra vérifier que toutes les variables aléatoires auxquelles on applique le barycentre 
prennent leurs valeurs dans un compact $K$ qui ne dépend que de la trajectoire de $X$ fixée. On utilisera donc la continuité de $\mathbf{B}$ évoquée à la remarque 2.4 .

Montrons la continuité de $X^{h}: t \mapsto X_{t}^{h}$. On écrit $X^{h}$ comme la composée $\mathbf{B} \circ \psi$ où

$$
\begin{aligned}
\psi: \mathbb{R}_{+} & \longrightarrow \mathcal{P} \\
t & \mapsto \mu_{t}^{h} \circ X^{-1} .
\end{aligned}
$$

L'application barycentre $\mathbf{B}$ étant continue le long de $\mu_{t}^{h} \circ X^{-1}$, il ne reste plus qu'à prouver la continuité de $\psi$ de $\mathbb{R}_{+}$dans $\mathcal{P}_{K}$ muni de la topologie de la convergence étroite.

Soit $f$ une fonction de $M$ dans $\mathbb{R}$ continue bornée et soient $s$ et $t$ dans $\mathbb{R}_{+}$:

$$
\begin{aligned}
\psi(t)(f)-\psi(s)(f) & =\frac{1}{h} \int_{t-h}^{t} f\left(X_{u}\right) \mathrm{d} u-\frac{1}{h} \int_{s-h}^{s} f\left(X_{u}\right) \mathrm{d} u \\
& =\frac{1}{h}\left(\int_{t-h}^{s-h} f\left(X_{u}\right) \mathrm{d} u+\int_{s}^{t} f\left(X_{u}\right) \mathrm{d} u\right)
\end{aligned}
$$

et donc

$$
|\psi(t)(f)-\psi(s)(f)| \leq \frac{2}{h}\|f\|_{\infty}|t-s| .
$$

Ce qui suffit à conclure.

Montrons que $X^{h}: t \mapsto X_{t}^{h}$ est à variation finie sur tout compact. Soit $T>0$ et soit $\left(t_{i}\right)_{1 \leq i \leq n}$ une subdivision de $[0, T]$ de pas strictement inférieur à $h$. Pour $i=1, \ldots, n-1$, on définit la variable aléatoire $\widetilde{X}^{i}$ sur $\left[t_{i}-h, t_{i}\right]$ par

$$
\tilde{X}_{s}^{i}= \begin{cases}X_{s+h} & \text { si } s \in\left[t_{i}-h, t_{i+1}-h[\right. \\ X_{s} & \text { si } s \in\left[t_{i+1}-h, t_{i}\right],\end{cases}
$$

ce qui donne l'égalité suivante entre lois :

$$
\mu_{t_{i+1}}^{h} \circ X^{-1}=\mu_{t_{i}}^{h} \circ\left(\tilde{X}^{i}\right)^{-1} .
$$

Cela permet alors d'estimer la distance entre $X_{t_{i+1}}^{h}$ et $X_{t_{i}}^{h}$ :

$$
\begin{aligned}
d_{M}\left(X_{t_{i+1}}^{h}, X_{t_{i}}^{h}\right)=d_{M}( & \left.\left.\mathbf{B}\left(\mu_{t_{i}}^{h} \circ\left(\tilde{X}^{i}\right)^{-1}\right), \mathbf{B}\left(\mu_{t_{i}}^{h} \circ X^{-1}\right)\right)\right) . \\
- & 242-
\end{aligned}
$$


D'après la propriété de Lipschitz sur $K$ du barycentre (définition 2.2b)),

$$
\begin{aligned}
& d_{M}\left(X_{t_{i+1}}^{h}, X_{t_{i}}^{h}\right) \leq \frac{C_{K}}{h} \int_{t_{i}-h}^{t_{i}} d_{M}\left(\tilde{X}_{s}^{i}, X_{s}\right) \mathrm{d} s \\
& d_{M}\left(X_{t_{i+1}}^{h}, X_{t_{i}}^{h}\right) \leq \frac{C_{K}}{h} \int_{t_{i}-h}^{t_{i+1}-h} d_{M}\left(X_{s+h}, X_{s}\right) \mathrm{d} s .
\end{aligned}
$$

Finalement,

$$
\sum_{i=1}^{n-1} d_{M}\left(X_{t_{i+1}}^{h}, X_{t_{i}}^{h}\right) \leq \frac{C_{K}}{h} \int_{0}^{T-h} d_{M}\left(X_{s+h}, X_{s}\right) \mathrm{d} s
$$

et comme, à trajectoire fixée, $s \mapsto X_{s}$ est bornée sur $[0, T]$, le terme de droite de cette dernière inégalité est fini.

(ii) Pour toute trajectoire fixée de $X$ et tout $t \in \mathbb{R}_{+}$, la convergence de $X_{t}^{h}=\mathbf{B}\left(\mu_{t}^{h} \circ X^{-1}\right)$ vers $X_{t}$ - quand $h$ tend vers 0 est immédiate en utilisant la continuité de $\mathbf{B}$ sur $\mathcal{P}_{K}$ et la convergence étroite de $\mu_{t}^{h} \circ X^{-1}$ vers la masse de Dirac $\delta_{X_{t^{-}}}$.

\subsection{Changement de temps}

Nous disposons maintenant d'une régularisation de la semi-martingale directrice $X$. Cependant comme dans le cas réel, la famille $\left(X^{h}\right)_{h>0}$ ne converge pas en loi (pour la topologie de Skorokhod) vers $X$, car les $X^{h}$ sont continus et $X$ ne l'est pas. Il n'est pas possible d'appliquer directement les résultats de [9] pour obtenir la convergence des solutions des équations différentielles associées. Kurtz [7] résout ce problème en introduisant des changements de temps qui ouvrent des intervalles de temps fictifs aux instants de sauts de $X$. Ce sont ces changements de temps que l'on rencontre à la première étape de [8] et que nous reprenons ici.

Dorénavant, la variété $M$ est plongée dans $\mathbb{R}^{m}$ et pour chaque élément $x$ de $M,\left(x^{1}, \ldots, x^{m}\right)$ désigne le système de coordonnées de $x$ dans $\mathbb{R}^{m}$. Ainsi la semi-martingale $X$, à valeurs dans $M$, a pour coordonnées $\left(X^{1}, \ldots, X^{m}\right)$ et on désigne par $[X, X]^{d}$ le processus croissant totalement discontinu :

$$
[X, X]_{t}^{d}=\sum_{0<s \leq t} d_{M}\left(X_{s}, X_{s^{-}}\right)^{2} .
$$

On définit pour tout $h>0$ et tout $t \in \mathbb{R}_{+}$

$$
\gamma_{h}(t)=\frac{1}{h} \int_{t-h}^{t}\left([X, X]_{s}^{d}+s\right) \mathrm{d} s
$$




\section{Serge Cohen et Anne Estrade}

ainsi que

$$
\gamma(t)=[X, X]_{t}^{d}+t
$$

Pour chaque $h>0, \gamma_{h}$ est une application continue strictement croissante, d'inverse notée $\gamma_{h}^{-1}$, tandis que $\gamma$ est strictement croissante càdlàg; on note $\gamma^{-1}$ son inverse continu :

$$
\gamma^{-1}(t)=\inf \{u>0 \mid \gamma(u)>t\}
$$

Remarque 2.9. - Il est intéressant de noter (cf. [8, lemme 6.1]) que :

(i) $\forall h>0, \forall t \geq 0, \gamma_{h}^{-1}(t)-h<\gamma^{-1}(t) \leq \gamma_{h}^{-1}(t)$,

(ii) $\left(\gamma_{h}^{-1}(t)\right)_{h>0}$ converge uniformément en $t$ vers $\gamma^{-1}(t)$ quand $h$ tend vers 0 .

Pour chaque $h>0$, nous effectuons le changement de temps $\gamma_{h}^{-1}$ sur la semi-martingale $X^{h}$. Soit $\hat{X}^{h}$ le processus obtenu par

$$
\widehat{X}_{t}^{h}=X^{h} \circ \gamma_{h}^{-1}(t)=\mathbf{B}\left(\mu_{\gamma_{h}^{-1}(t)}^{h} \circ X^{-1}\right)
$$

qui est, comme $X^{h}$, continue à variation finie sur tout compact. Comme $\widehat{X}^{h}$ est adapté à la filtration $\left(\mathcal{F}_{\gamma_{h}^{-1}(t)}\right)_{t}$, c'est une semi-martingale pour cette filtration.

Pour tout $t \in \mathbb{R}_{+}$, nous nous intéressons à la convergence (éventuelle) de $\widehat{X}_{t}^{h}$ quand $h$ tend vers $0^{+}$. On introduit pour cela les instants suivants :

$$
\begin{aligned}
& \eta_{1}(t)=\sup \left\{s \mid \gamma^{-1}(s)<\gamma^{-1}(t)\right\} \\
& \eta_{2}(t)=\inf \left\{s \mid \gamma^{-1}(s)>\gamma^{-1}(t)\right\}
\end{aligned}
$$

\section{Remarquons que}

- si $\gamma^{-1}(t)$ n'est pas un instant de saut de $X$, alors $\eta_{1}(t)=\eta_{2}(t)=t$,

- si $\gamma^{-1}(t)$ est un instant de saut de $X$, alors $\eta_{1}(t)<\eta_{2}(t), t \in$ $\left[\eta_{1}(t), \eta_{2}(t)\right]$ et $\eta_{2}(t)-\eta_{1}(t)=[X, X]_{\gamma^{-1}(t)}^{d}-[X, X]_{\gamma^{-1}(t)^{-}}^{d}$.

Nous allons prouver un résultat de convergence analogue au lemme 6.2 de [8]. 
Régularisation d'équations de Stratonovitch à sauts entre variétés

Proposition 2.10. - La famille de processus $\left(\widehat{X}^{h}\right)_{h>0}$ converge presque sûrement, uniformément sur tout compact, vers le processus $\widehat{X}$ donné par

$$
\widehat{X}_{t}= \begin{cases}X \circ \gamma^{-1}(t) & \text { si } \eta_{1}(t)=\eta_{2}(t) \\ \mathbf{B}\left(\zeta_{t}\right) & \text { si } \eta_{1}(t)<\eta_{2}(t)\end{cases}
$$

où $\zeta_{t}$ est la mesure de probabilité sur $M$ définie, pour $\eta_{1}(t)<\eta_{2}(t)$, par

$$
\zeta_{t}=\frac{\eta_{2}(t)-t}{\eta_{2}(t)-\eta_{1}(t)} \delta_{X_{\gamma^{-1}(t)-}}+\frac{t-\eta_{1}(t)}{\eta_{2}(t)-\eta_{1}(t)} \delta_{X_{\gamma^{-1}(t)}} .
$$

Preuve. - Soit $T>0$. On suppose fixée une trajectoire de $X$ entre 0 et $T$, qui est alors contenue dans un compact fixe $K$ de $M$.

Notons encore $\zeta_{t}$ la mesure de probabilité sur $M$ définie par (10) pour $\eta_{1}(t)<\eta_{2}(t)$ et par $\zeta_{t}=\delta_{X_{\gamma^{-1}(t)}}$ pour $\eta_{1}(t)=\eta_{2}(t)$. Nous devons alors prouver que $\mathbf{B}\left(\mu_{\gamma_{h}^{-1}(t)}^{h} \circ X^{-1}\right)$ tend vers $\mathbf{B}\left(\zeta_{t}\right)$ uniformément pour $t \in[0, T]$. En utilisant la continuité de $\mathbf{B}$ sur $\mathcal{P}_{K}$, cela revient à montrer que pour toute famille $\left(t_{h}\right)_{h>0}$ de réels dans $[0, T]$ convergeant vers $t$, $\mu_{\gamma_{h}^{-1}\left(t_{h}\right)}^{h} \circ X^{-1}$ converge étroitement vers $\zeta_{t}$.

Soit $\left(t_{h}\right)_{h>0}$ une famille de réels convergeant vers un réel fixe $t$. Pour $f$ de $M$ dans $\mathbb{R}$ continue bornée,

$$
\mu_{\gamma_{h}^{-1}\left(t_{h}\right)}^{h} \circ X^{-1}(f)=\frac{1}{h} \int_{\gamma_{h}^{-1}\left(t_{h}\right)-h}^{\gamma_{h}^{-1}\left(t_{h}\right)} f\left(X_{s}\right) \mathrm{d} s .
$$

- Si $\eta_{1}(t)=\eta_{2}(t)$, alors $\gamma^{-1}(t)$ est un instant de continuité de $X$ et d'après le (ii) de la remarque $2.9, \mu_{\gamma_{h}^{-1}\left(t_{h}\right)}^{h} \circ X^{-1}(f)$ converge alors vers $f\left(X_{\gamma^{-1}(t)}\right)$, ce qui, dans ce cas, n'est rien d'autre que $\zeta_{t}(f)$.

- Si $\eta_{1}(t)<\eta_{2}(t)$, plusieurs cas se présentent suivant la position des $\gamma_{h}^{-1}\left(t_{h}\right)$ par rapport à $\gamma^{-1}(t)$.

Pour les sous-suites telles que $\gamma_{h}^{-1}\left(t_{h}\right) \leq \gamma^{-1}(t)$ pour tout $h>0$, il est clair que $\mu_{\gamma_{h}^{-1}\left(t_{h}\right)}^{h} \circ X^{-1}(f)$ converge vers $f\left(X_{\left.\gamma^{-1}(t)-\right)}\right.$. D'autre part, s'il existe une telle sous-suite, alors $t_{h} \leq \gamma_{h} \circ \gamma^{-1}(t)$ pour tout $h>0$, et donc, en passant à la limite, $t \leq \eta_{1}(t)$, soit encore $t=\eta_{1}(t)$. Dans ces conditions, d'après $(10), f\left(X_{\gamma^{-1}(t)^{-}}\right)=\zeta_{t}(f)$. 


\section{Serge Coher, et Anne Estrade}

Pour les sous-suites telles que $\gamma_{h}^{-1}\left(t_{h}\right) \geq \gamma^{-1}(t)+h$ pour tout $h>0$, des arguments similaires mènent à l'égalité $t=\eta_{2}(t)$ et à la convergence de $\mu_{\gamma_{h}^{-1}\left(t_{h}\right)}^{h} \circ X^{-1}(f)$ vers $f\left(X_{\gamma^{-1}(t)}\right)=\zeta_{t}(f)$. Notons qu'il ne peut exister simultanément une sous-suite de ce type et une sous-suite du type précédent car $\eta_{1}(t) \neq \eta_{2}(t)$.

Enfin, pour les sous-suites telles que $\gamma^{-1}(t)<\gamma_{h}^{-1}\left(t_{h}\right)<\gamma^{-1}(t)+h$ pour tout $h>0$, on découpe l'intervalle $\left[\gamma_{h}^{-1}\left(t_{h}\right)-h, \gamma_{h}^{-1}\left(t_{h}\right)\right]$ en deux parties au point $\gamma^{-1}(t)$. Ceci donne

$$
\begin{aligned}
\mu_{\gamma_{h}^{-1}\left(t_{h}\right)}^{h} \circ X^{-1}(f)= & \frac{1}{h} \int_{\gamma^{-1}(t)-\left(1-q\left(h, t_{h}\right)\right) h}^{\gamma^{-1}(t)} f\left(X_{s}\right) \mathrm{d} s+ \\
& +\frac{1}{h} \int_{\gamma^{-1}(t)}^{\gamma^{-1}(t)+q\left(h, t_{h}\right) h} f\left(X_{s}\right) \mathrm{d} s
\end{aligned}
$$

en notant

$$
q(h, s)=\frac{1}{h}\left(\cdot{ }^{-1}(s)-\gamma^{-1}(t)\right) .
$$

Nous allons montrer que $q\left(h, t_{h}\right)$ tend vers $t-\eta_{1}(t) /\left(\eta_{2}(t)-\eta_{1}(t)\right)$.

En remarquant que $q\left(h, \gamma_{h} \circ \gamma^{-1}(t)\right)=0$, on écrit

$$
q\left(h, t_{h}\right)=\int_{\gamma_{h} \circ \gamma^{-1}(t)}^{t_{h}} \frac{\partial q}{\partial s}(h, s) \mathrm{d} s
$$

et d'après la définition (7) de $\gamma_{h}$,

$$
\frac{\partial q}{\partial s}(h, s)=\frac{1}{h} \frac{\mathrm{d} \gamma_{h}^{-1}(s)}{\mathrm{d} s}=\frac{1}{[X, X]_{\gamma_{h}^{-1}(s)}^{d}-[X, X]_{\gamma_{h}^{-1}(s)-h}^{d}+h} .
$$

Pour les $s$ tels que $\gamma^{-1}(t)<\gamma_{h}^{-1}(s)<\gamma^{-1}(t)+h$ pour tout $h>0$, $\partial q(h, s) / \partial s$ converge uniformément en $s$ vers

$$
\frac{1}{[X, X]_{\gamma^{-1}(t)}^{d}-[X, X]_{\gamma^{-1}(t)^{-}}^{d}}=\frac{1}{\eta_{2}(t)-\eta_{1}(t)} .
$$

Par suite, en utilisant une nouvelle fois que $\gamma_{h} \circ \gamma^{-1}(t)$ tend vers $\eta_{1}(t)$, on obtient

$$
\lim _{h \rightarrow 0} q\left(h, t_{h}\right)=\int_{\eta_{1}(t)}^{t} \frac{1}{\eta_{2}(t)-\eta_{1}(t)} \mathrm{d} s=\frac{t-\eta_{1}(t)}{\eta_{2}(t)-\eta_{1}(t)}
$$


Finalement, en faisant tendre $h$ vers 0 , l'égalité (11) fournit la limite de $\mu_{\gamma_{h}^{-1}(t)}^{h} \circ X^{-1}(f)$ :

$$
\left(1-\frac{t-\eta_{1}(t)}{\eta_{2}(t)-\eta_{1}(t)}\right) f\left(X_{\gamma^{-1}(t)^{-}}\right)+\frac{t-\eta_{1}(t)}{\eta_{2}(t)-\eta_{1}(t)} f\left(X_{\gamma^{-1}(t)}\right) .
$$

Ceci est égal à $\zeta_{t}(f)$ d'après $(10)$ et termine la preuve.

\subsection{Un bon processus : $A^{h}$}

La convergence des $\widehat{X}^{h}$ ne suffit pas à entraîner la convergence des solutions d'EDS dirigées par $\widehat{X}^{h}$. Pour obtenir cette convergence, nous avons besoin d'une notion plus forte. Nous empruntons à [9] la définition suivante.

DÉfinition 2.11. - Soit $\left(Z^{h}\right)_{h>0}$ une famille de processus telle que, $\forall h>0, Z^{h}$ est une semi-martingale adaptée à une filtration $\left(\mathcal{F}_{t}^{h}\right)_{t \geq 0}$ et telle que, quand $h$ tend vers $0, Z^{h}$ converge en loi pour la topologie de Skorokhod vers un processus $Z$. On dit que la famille $\left(Z^{h}\right)_{h>0}$ est bonne si $Z$ est une semi-martingale et si pour tout processus $H^{h}$ càdlàg adapté à $\mathcal{F}^{h}$, la convergence en loi pour la topologie de Skorokhod de $\left(H^{h}, Z^{h}\right)$ vers $(H, Z)$ entraine la convergence en loi pour la topologie de Skorokhod de $\int_{0}^{\cdot} H_{s^{-}}^{h} \mathrm{~d} Z_{s}^{h}$ vers $\int_{0}^{\cdot} H_{s^{-}} \mathrm{d} Z_{s}$.

Plusieurs auteurs se sont intéressés à cette notion et ont donné des critères pour qu'une famille de semi-martingales soit bonne (critère "UT" pour Jakubowski, Mémin et Pagès dans [6]; condition C2.2(i) pour Kurtz et Protter dans [9] par exemple).

Pour les processus qui nous concernent, la famille $\left(X \circ \gamma_{h}^{-1}\right)_{h>0}$ est bonne; en effet, $\gamma_{h}^{-1}$ converge uniformément vers $\gamma^{-1}$ par valeur supérieures (remarque 2.9), donc $X \circ \gamma_{h}^{-1}$ converge uniformément vers $X \circ \gamma^{-1}$, qui de plus est une semi-martingale. En revanche, la famille $\left(\widehat{X}^{h}\right)_{h>0}$ n'est pas bonne car elle converge vers $\widehat{X}$ qui n'est pas nécessairement une semimartingale. Aussi, nous faut-il introduire de nouveaux processus définis par : pour tout $h>0, t \geq 0, i, j \in\{1, \ldots, m\}$

$$
\begin{aligned}
R_{t}^{h, i}= & \widehat{X}_{t}^{h, i}-X_{\gamma_{h}^{-1}(t)}^{i} \\
A_{t}^{h(i, j)}= & \int_{0}^{t} R_{s}^{h, i} \mathrm{~d} \widehat{X}_{s}^{h, j} . \\
& -247-
\end{aligned}
$$




\section{Serge Cohen et Anne Estrade}

Comme les $\widehat{X}^{h}$, les $A^{h}$ sont à variation finie sur tout compact et sont des semi-martingales pour les filtrations $\left(\mathcal{F}_{\gamma_{h}^{-1}(t)}\right)_{t}$, mais les $A^{h}$, eux, constituent une bonne famille. Le lemme suivant établit cette propriété.

LEMME 2.12. - La famille des semi-martingales $\left(A^{h}\right)_{h>0}$ est une bonne famille.

Preuve. - Pour les processus à variation finie, il est relativement simple de prouver la condition C2.2(i) de [9]. Il suffit par exemple de montrer que sur tout compact, les variations sont bornées dans $L^{1}$ uniformément en $h$.

Soit $T>0$. Par changement de variables, on obtient :

$$
\mathbf{E} \int_{0}^{T}\left|d A_{t}^{h(i, j)}\right| \leq \mathbf{E} \int_{0}^{\gamma_{h}^{-1}(T)}\left|X_{s}^{h, i}-X_{s}^{i}\right|\left|\mathrm{d} X_{s}^{h, j}\right|
$$

On peut supposer les trajectoires de la semi-martingale $X$, entre 0 et $T$, contenues presque sûrement dans un compact fixe $K$ de la variété $M$.

Majorons d'abord l'intégrant $\left|X_{s}^{h, i}-X_{s}^{i}\right|$ de (13). Les applications coordonnées restreintes à $K$ étant $k$-lipschitziennes, $\forall s \in[0, T]$,

$$
\left|X_{s}^{h, i}-X_{s}^{i}\right| \leq k d_{M}\left(X_{s}^{h}, X_{s}\right) .
$$

En écrivant $X_{s}^{h}=\mathbf{B}\left(\mu_{s}^{h} \circ X^{-1}\right)$ et $X_{s}=\mathbf{B}\left(\mu_{s}^{h} \circ X_{s}^{-1}\right)$, et en utilisant la propriété de Lipschitz du barycentre $\mathbf{B}$, on obtient

$$
\begin{aligned}
\left|X_{s}^{h, i}-X_{s}^{i}\right| & \leq k C_{K} \frac{1}{h} \int_{s-h}^{s} d_{M}\left(X_{u}, X_{s}\right) \mathrm{d} u \\
& \leq 2 k C_{K} \sup _{u \in[s-h, s]} d_{M}\left(X_{u}, X_{s-h}\right) .
\end{aligned}
$$

Majorons ensuite l'intégrateur $\left|\mathrm{d} X_{s}^{h, j}\right|$ de (13). Pour $t>s$, le caractère lipschitzien des applications coordonnées sur $K$ donne

$$
\frac{\left|X_{t}^{h, i}-X_{s}^{h, i}\right|}{t-s} \leq k \frac{d_{M}\left(X_{t}^{h}, X_{s}^{h}\right)}{t-s},
$$

et en se souvenant de l'inégalité (6), on obtient

$$
\begin{aligned}
\frac{\left|X_{t}^{h, i}-X_{s}^{h, i}\right|}{t-s} \leq \frac{k C_{K}}{h(t-s)} \int_{[s-h, t-h]} d_{M}\left(X_{u+h}, X_{u}\right) \mathrm{d} u . \\
-248-
\end{aligned}
$$


Régularisation d'équations de Stratonovitch à sauts entre variétés

Quand $t$ tend vers $s$ par valeurs supérieures, le terme de droite tend vers $\left(k C_{K} / h\right) d_{M}\left(X_{s}, X_{s-h}\right)$. Par suite, pour toute fonction mesurable bornée $f$,

$$
\int_{0}|f(s)|\left|\mathrm{d} X_{s}^{h, i}\right| \leq \frac{k C_{K}}{h} \int_{0}|f(s)| d_{M}\left(X_{s}, X_{s-h}\right) \mathrm{d} s
$$

et donc

$$
\int_{0}|f(s)|\left|\mathrm{d} X_{s}^{h, i}\right| \leq \frac{k C_{K}}{h} \int_{0}|f(s)| \sup _{u \in[s-h, s]} d_{M}\left(X_{u}, X_{s-h}\right) \mathrm{d} s .
$$

Finalement, si $h \leq 1$,

$$
\int_{0}^{T}\left|\mathrm{~d} A_{t}^{h(i, j)}\right| \leq \frac{2 k^{2} C_{K}^{2}}{h} \int_{0}^{\gamma^{-1}(T)+1} \sup _{u \in[s-h, s]} d_{M}\left(X_{u}, X_{s-h}\right)^{2} \mathrm{~d} s .
$$

On conclut alors comme dans le lemme 6.3 de [8] : à l'aide de l'inégalité ci-dessus, on majore $\mathbf{E}_{\mathbf{Q}} \int_{0}^{T}\left|\mathrm{~d} A_{t}^{h(i, j)}\right| \operatorname{par} C\|X\|_{H^{2}}$, indépendamment de $h$ pour une probabilité $\mathbf{Q}$ équivalente à $\mathbf{P}$ sous laquelle la semi-martingale vectorielle $X=\left(X^{1}, \ldots, X^{m}\right)$ est dans $\mathcal{H}^{2}$. Un tel changement de probabilité n'affecte pas le caractère "bon" d'une famille de semi-martingales quand la convergence est une convergence en probabilité.

Une fois prouvée cette bonne propriété, se pose naturellement la question de la convergence des $A^{h}$ quand $h$ tend vers zéro. Les lemmes suivants apportent une réponse partielle à cette question; l'étude au voisinage des sauts éventuels de $X$ est reportée au moment de la démonstration générale. Nous établissons cette convergence en deux étapes : d'abord dans un cadre vectoriel en prenant l'espérance comme barycentre, puis dans le cadre géométrique d'une variété plongée.

LEMME 2.13. - Soit $X=\left(X^{i}\right)_{1 \leq i \leq m}$ une semi-martingale vectorielle continue et soit $\left(X^{h}\right)_{h>0}$ la famille des semi-martingales régularisées définies par

$$
X_{t}^{h, i}=\frac{1}{h} \int_{t-h}^{t} X_{s}^{i} \mathrm{~d} s .
$$

Alors, pour $i, j=1, \ldots, m$, la famille $\left(A^{h(i, j)}\right)_{h>0}$ donnée par (12) converge uniformément sur tout compact en probabilité (ucp) vers $-(1 / 2)\left[X^{i}, X^{j}\right]_{\gamma^{-1}}$ quand $h$ tend vers 0 . 


\section{Serge Cohen et Anne Estrade}

Preuve. - Dans ce cadre vectoriel continu, l'expression des processus $X^{h, i}$ nous conduit à utiliser des résultats d'approximation d'intégrales établis par Russo et Vallois [12] qui s'écrivent ainsi : quand $h$ tend vers zéro,

$$
\begin{aligned}
& \text { (A1) }\left(\frac{1}{h} \int_{0}^{\cdot} X_{s}^{i}\left(X_{s+h}^{j}-X_{s}^{j}\right) \mathrm{d} s\right)_{h>0} \text { converge ucp vers } \int_{0}^{\cdot} X_{s}^{i} \mathrm{~d} X_{s}^{j} \\
& \text { (A2) }\left(\frac{1}{h} \int_{0}^{\cdot} X_{s}^{i}\left(X_{s}^{j}-X_{s-h}^{j}\right) \mathrm{d} s\right)_{h>0} \text { converge ucp vers } \int_{0}^{\cdot} X_{s}^{i} \mathrm{~d} X_{s}^{j}+ \\
& {\left[X^{i}, X^{j}\right] ;}
\end{aligned}
$$

où la convergence ucp désigne la convergence uniforme sur tout compact en probabilité.

De (12), on déduit par un changement de variable,

$$
A_{t}^{h(i, j)}=\int_{0}^{\gamma_{h}^{-1}(t)}\left(X_{s}^{h, i}-X_{s}^{i}\right) \mathrm{d} X_{s}^{h, j} .
$$

Les convergences obtenues sont uniformes sur tout compact, donc $A_{t}^{h(i, j)}$ aura la même limite que $B_{\gamma^{-1}(t)}^{h(i, j)}$ où

$$
B_{t}^{h(i, j)}=\int_{0}^{t}\left(X_{s}^{h, i}-X_{s}^{i}\right) \mathrm{d} X_{s}^{h, j} .
$$

En se rappelant la définition des $X^{h, i}$, on obtient

$$
\begin{aligned}
B_{t}^{h(i, j)} & =\frac{1}{h} \int_{0}^{t}\left(\int_{s-h}^{s}\left(X_{u}^{i}-X_{s}^{i}\right) \frac{\mathrm{d} u}{h}\right)\left(X_{s}^{j}-X_{s-h}^{j}\right) \mathrm{d} s \\
& =\frac{1}{h} \int_{0}^{t}\left(\int_{0}^{1}\left(X_{s-v h}^{i}-X_{s}^{i}\right) \mathrm{d} v\right)\left(X_{s}^{j}-X_{s-h}^{j}\right) \mathrm{d} s \\
& =\int_{0}^{1} F_{t}^{h}(v) \mathrm{d} v
\end{aligned}
$$

où on a noté, pour tout $v \in[0,1]$,

$$
\begin{aligned}
F_{t}^{h}(v)= & \frac{1}{h} \int_{0}^{t}\left(X_{s-v h}^{i}-X_{s}^{i}\right)\left(X_{s}^{j}-X_{s-h}^{j}\right) \mathrm{d} s \\
= & \frac{1}{h} \int_{0}^{t} X_{s-v h}^{i}\left(X_{s}^{j}-X_{s-v h}^{j}\right) \mathrm{d} s+ \\
& +\frac{1}{h} \int_{0}^{t} X_{s-v h}^{i}\left(X_{s-v h}^{j}-X_{s-h}^{j}\right) \mathrm{d} s-\frac{1}{h} \int_{0}^{t} X_{s}^{i}\left(X_{s}^{j}-X_{s-h}^{j}\right) \mathrm{d} s \\
= & F_{t}^{h(1)}(v)+F_{t}^{h(2)}(v)+F_{t}^{h(3)}(v) . \\
& -250-
\end{aligned}
$$


Le premier terme de cette somme s'écrit

$$
F_{t}^{h(1)}(v)=\frac{1}{h} \int_{0}^{t-v h} X_{s}^{i}\left(X_{s+v h}^{j}-X_{s}^{j}\right) \mathrm{d} s
$$

et, d'après (A1) et la continuité de $X$, converge ucp vers

$$
F_{t}^{1}(v)=v \int_{0}^{t} X_{s}^{i} \mathrm{~d} X_{s}^{j}
$$

Une transformation similaire du second terme $F_{t}^{h(2)}(v)$ et l'argument (A2) prouvent la convergence ucp de $F_{t}^{h(2)}(v)$ vers

$$
F_{t}^{2}(v)=(1-v)\left(\int_{0}^{t} X_{s}^{i} \mathrm{~d} X_{s}^{j}+\left[X^{i}, X^{j}\right]_{t}\right)
$$

Enfin, le troisième terme $F_{t}^{h(3)}(v)$, qui est indépendant de $v$, converge ucp vers

$$
F_{t}^{3}(v)=-\int_{0}^{t} X_{s}^{i} \mathrm{~d} X_{s}^{j}-\left[X^{i}, X^{j}\right]_{t}
$$

Finalement, pour tout $v \in[0,1], F_{t}^{h}(v)$ converge vers $-v\left[X^{i}, X^{j}\right]_{t}$, et par convergence dominée, en intégrant $v$ entre 0 et 1 , on obtient la convergence ucp de $B_{t}^{h(i, j)}$, donc de $A_{t}^{h(i, j)}$, vers $-(1 / 2)\left[X^{i}, X^{j}\right]_{\gamma^{-1}(t)}$.

La convergence des $A^{h}$ pour un processus $X$ réel continu figure dans [8]; la preuve repose uniquement sur une intégration par parties et ne nécessite pas de faire appel aux approximations de [12]. Le lemme 2.13 et les propositions qui suivent montrent que les résultats de [8] demeurent sans changement dans le cas où $X$ est vectoriel. Cette remarque n'est pas anodine; nous renvoyons à [1] pour une discussion générale sur les différentes formes d'approximations de semi-martingales vectorielles.

Revenons maintenant à la semi-martingale $X$ à valeurs dans la variété $M$ et notons de nouveau $\left(X^{i}\right)_{1 \leq i \leq m}$ la semi-martingale vectorielle issue du plongement de la variété dans $\mathbb{R}^{m}$. On désigne par $\left(X^{h}\right)_{h}$ la famille des régularisées de $X$ donnée par (5). Le lemme suivant montre que les $A^{h}$ restreints aux instants où $X$ est continu convergent vers le crochet changé de temps de la partie martingale continue de $X$. 


\section{Serge Cohen et Anne Estrade}

LEMme 2.14. - Soient $\left(\tau_{l}\right)_{\ell \geq 1}$ les instants de sauts de $X$. On note

$$
\Gamma=\bigcup_{\ell \geq 1}\left[\gamma\left(\tau_{\ell}^{-}\right), \gamma\left(\tau_{\ell}\right)\right] \text { et } \Gamma^{c}=\mathbb{R}^{+} \backslash \Gamma \text {. }
$$

Pour $i, j=1, \ldots, m$, la famille $\left(\int_{0}^{t} 1_{\Gamma^{c}}(s) \mathrm{d} A_{s}^{h(i, j)}\right)_{h>0}$ où $A^{h(i, j)}$ est donné par (12) converge uniformément sur tout compact en probabilité vers $-(1 / 2)\left\langle\left(X^{i}\right)^{c},\left(X^{j}\right)^{c}\right\rangle_{\gamma^{-1}(t)}$ quand $h$ tend vers zéro.

Preuve. - Rappelons que, pour tout $h>0,\left(X^{h, i}\right)_{1 \leq i<m}$ désigne la semi-martingale vectorielle obtenue à partir de $X$ par régularisation dans $M$ puis plongement dans $\mathbb{R}^{m}$, et notons $\left(\tilde{X}^{h, i}\right)_{1 \leq i \leq m}$ la semi-martingale vectorielle obtenue à partir de $X$ par plongement dans $\mathbb{R}^{m}$ puis régularisation.

Les lemmes 2.12 et 2.13 assurent la convergence de

$$
\int_{0}^{\gamma_{h}^{-1}(t)} 1_{\Gamma^{c}}(s)\left(\tilde{X}_{s}^{h, i}-X_{s}^{i}\right) \mathrm{d} \tilde{X}_{s}^{h, j}
$$

vers

$$
-\frac{1}{2} \int_{0}^{t} 1_{\Gamma^{c}}(s) \mathrm{d}\left[X^{i}, X^{j}\right]_{\gamma^{-1}(s)}=-\frac{1}{2}\left\langle\left(X^{i}\right)^{c},\left(X^{j}\right)^{c}\right\rangle_{\gamma^{-1}(t)}
$$

et il s'agit maintenant de montrer que la différence $\Delta^{h(i, j)}$ entre les variations sur $\Gamma^{c} \mathrm{du}$ " $A^{h(i, j)}$ vectoriel" et celles du " $A^{h(i, j)}$ de la variété" tend vers zéro.

En reprenant les notations introduites au début de ce paragraphe, on a $X_{s}^{h}=\mathbf{B}\left(\mu_{s}^{h} \circ X^{-1}\right)$. Comme, pour $i=1, \ldots, m, \tilde{X}_{s}^{h, i}=(1 / h) \int_{s-h}^{s} X_{u}^{i} \mathrm{~d} u$, $\tilde{X}_{s}^{h}$ peut être vu, à $\omega$ fixé, comme l'espérance du vecteur aléatoire $\left(X^{i}(\omega)\right)_{i}$ défini sur l'espace de probabilité $\left([s-h, s], \mu_{s}^{h}\right)$, que nous notons $\mathbf{E}_{\mu_{s}^{h}}(X)$. De même, $\operatorname{var}_{\mu_{s}^{h}}(X)$ désigne la variance de cette variable aléatoire. La propriété de Lipschitz des applications coordonnées et la condition c) de la définition $2.2 \mathrm{du}$ barycentre permettent d'écrire successivement :

$$
\begin{aligned}
\left|X_{s}^{h, i}-\tilde{X}_{s}^{h, i}\right| & \leq k\left|\mathbf{B}\left(\mu_{s}^{h} \circ X^{-1}\right)-\mathbf{E}_{\mu_{s}^{h}}(X)\right| \\
& \leq k C \operatorname{var}_{\mu_{s}^{h}}(X) \\
& \leq k C \inf _{x \in M}\left(\frac{1}{h} \int_{s-h}^{s} d_{M}\left(X_{u}, x\right)^{2} \mathrm{~d} u\right) \\
& \leq k C \inf _{x \in M}\left(\sup _{u \in[s-h, s]} d_{M}\left(X_{u}, x\right)^{2}\right) .
\end{aligned}
$$


Régularisation d'équations de Stratonovitch à sauts entre variétés

Finalement

$$
\left|X_{s}^{h, i}-\tilde{X}_{s}^{h, i}\right| \leq k C \sup _{u \in[s-h, s]} d_{M}\left(X_{u}, X_{s}\right)^{2}
$$

On regarde maintenant la différence $\Delta_{t}^{h(i, j)}$ :

$$
\begin{aligned}
\Delta_{t}^{h(i, j)}= & \int_{0}^{\gamma_{t}^{-1}} \mathbf{1}_{\Gamma^{c}}(s)\left(\tilde{X}_{s}^{h, i}-X_{s}^{i}\right) \mathrm{d} \tilde{X}_{s}^{h, j}-\int_{0}^{\gamma_{t}^{-1}} \mathbf{1}_{\Gamma^{c}}(s)\left(X_{s}^{h, i}-X_{s}^{i}\right) \mathrm{d} X_{s}^{h, j} \\
= & \int_{0}^{\gamma_{t}^{-1}} \mathbf{1}_{\Gamma^{c}}(s)\left(\tilde{X}_{s}^{h, i}-X_{s}^{h, i}\right) \mathrm{d} \tilde{X}_{s}^{h, j}+ \\
& +\int_{0}^{\gamma_{t}^{-1}} \mathbf{1}_{\Gamma^{c}}(s)\left(X_{s}^{h, i}-X_{s}^{i}\right) \mathrm{d}\left(\tilde{X}_{s}^{h, j}-X_{s}^{h, j}\right) \\
= & \int_{0}^{\gamma_{t}^{-1}} \mathbf{1}_{\Gamma^{c}}(s)\left(\tilde{X}_{s}^{h, i}-X_{s}^{h, i}\right) \mathrm{d} \tilde{X}_{s}^{h, j}+ \\
& +\int_{0}^{\gamma_{t}^{-1}} \mathbf{1}_{\Gamma^{c}}(s) \mathrm{d}\left(\left(X_{t}^{h, i}-X_{t}^{i}\right)\left(\tilde{X}_{t}^{h, j}-X_{t}^{h, j}\right)\right)+ \\
& -\int_{0}^{\gamma_{t}^{-1}} \mathbf{1}_{\Gamma^{c}}(s)\left(\tilde{X}_{s}^{h, j}-X_{s}^{h, j}\right) \mathrm{d} X_{s}^{h, i}+ \\
& +\int_{0}^{\gamma_{t}^{-1}} \mathbf{1}_{\Gamma^{c}}(s)\left(\tilde{X}_{s}^{h, j}-X_{s}^{h, j}\right) \mathrm{d} X_{s}^{i} \\
= & \Delta_{t}^{h(1)}+\Delta_{t}^{h(2)}+\Delta_{t}^{h(3)}+\Delta_{t}^{h(4)}
\end{aligned}
$$

Le terme $\Delta_{t}^{h(4)}$ tend vers 0 p.s. d'après le lemme 2.8. Les autres termes se traitent grâce à l'inégalité (15) ci-dessus et l'inégalité (14) qui s'appliquent autant à $\mathrm{d} X^{h, i}$ qu'à $\mathrm{d} \tilde{X}^{h, j}$. Ainsi,

$$
\begin{aligned}
& \left|\Delta_{t}^{h(1)}\right|+\left|\Delta_{t}^{h(2)}\right|+\left|\Delta_{t}^{h(3)}\right| \leq \\
& \quad \leq 3 k^{2} C C_{K} \frac{1}{h} \int_{0}^{\gamma_{t}^{-1}} 1_{\Gamma^{c}}(s) \sup _{u \in[s-h, s]} d_{M}\left(X_{u}, X_{s}\right)^{3} \mathrm{~d} s
\end{aligned}
$$

et par le même argument que dans le lemme 2.12, on obtient la convergence de ces termes vers 0 uniformément en probabilité. 


\section{Régularisation d'équations différentielles entre variétés}

\section{1 Équations entre variétés}

Puisque le processus directeur $X$ de notre étude est à valeurs dans une variété, il faut rappeler comment on peut présenter une équation différentielle entre des variétés $M$ et $N$. Dans le cas des équations ordinaires, on décrit le coefficient par une famille d'opérateurs linéaires $\{e(x, y)\}_{x \in M, y \in N}$ où $e(x, y)$ va de $T_{x} M$ dans $T_{y} N$, l'application $e$ étant supposée de classe $C^{3}$ au moins avec des dérivées bor lées (cette régularité est imposée par le calcul de Stratonovitch ultérieur). Iinsi l'équation

$$
\frac{\mathrm{d}}{\mathrm{d} t} y_{t}=e\left(x_{t}, y_{t}\right) ;-x_{t}, \quad y(0)=y_{0} \in N
$$

possède une signification intrinsèque quand on suppose que $x_{t}$ est un chemin $C^{1}$ sur $M$ et que la solution $y_{t}$ est de classe $C^{1}$ à valeurs dans $N$. En utilisant deux plongements $\left(x^{i}\right)_{i=1, \ldots, m},\left(y^{\alpha}\right)_{\alpha=1, \ldots, n}$ de classe $C^{3}$ au moins de $M$ dans $\mathbb{R}^{m}$, respectivement de $N$ dans $\mathbb{R}^{n}$ (ces notations seront conservées pour la présentation des systèmes différentiels à venir), le système d'équations

$$
\mathrm{d} Y_{t}^{\alpha}=e_{i}^{\alpha}\left(X_{t}, Y_{t}\right) \mathrm{d} X_{t}^{i}, \quad Y^{\alpha}(0)=Y_{0}^{\alpha}
$$

est une généralisation immédiate de (16) quand $X$ est un processus continu à variation finie. Ceci s'étend aux équations différentielles de Stratonovitch dirigées par des semi-martingales continues ou non, notion présentée dans [3] qui est une extension de [8] au calcul sur les variétés. Les coefficients de telles équations sont obtenus par un procédé analogue au cas vectoriel grâce au flot $\varphi(\cdot, x, y, z)$ de l'équation

$$
\left\{\begin{array}{l}
\frac{\mathrm{d}}{\mathrm{d} t} y_{t}=e(I(t, x, z), y(t)) \frac{\mathrm{d}}{\mathrm{d} t}(I(t, x, z)) \\
y(0)=y
\end{array}\right.
$$

où $I$ est un interpolateur. On définit ainsi une application $\varphi$ de $M \times N \times M$ dans $N$

$$
\begin{aligned}
& \varphi(x, y, z) \stackrel{\text { def }}{=} \varphi(1, x, y, z) \\
&-254-
\end{aligned}
$$


qui permet d'écrire une EDS entre variétés grâce au système différentiel

$$
\begin{aligned}
Y^{\alpha}= & y_{0}^{\alpha}+\int_{0} \frac{\partial \varphi^{\alpha}}{\partial z^{i}}\left(X_{s^{-}}, Y_{s^{-}}, X_{s^{-}}\right) \mathrm{d} X_{s}^{i} \\
& +\frac{1}{2} \int_{0} \frac{\partial^{2} \varphi^{\alpha}}{\partial z^{i} \partial z^{j}}\left(X_{s^{-}}, Y_{s^{-}}, X_{s^{-}}\right) \mathrm{d}\left\langle\left(X^{i}\right)^{c},\left(X^{j}\right)^{c}\right\rangle_{s} \\
& +\sum_{0<s \leq .}\left(\varphi^{\alpha}\left(X_{s^{-}}, Y_{s^{-}}, X_{s}\right)-Y_{s^{-}}^{\alpha}-\frac{\partial \varphi^{\alpha}}{\partial z^{i}}\left(X_{s^{-}}, Y_{s^{-}}, X_{s^{-}}\right) \Delta X_{s}^{i}\right) .
\end{aligned}
$$

Dans la formule précédente, $\partial / \partial z^{i}$ indique une dérivation partielle par rapport à la $i$-ème coordonnée de la dernière variable de $\varphi$; cette convention demeure dans la suite de l'article. On vérifie que $Y$ est une semi-martingale à valeurs dans $N$ et le caractère intrinsèque de (19) dans [2].

Remarque 3.1. - Un calcul élémentaire (cf. [3]) de dérivation du flot $\varphi$ par rapport aux paramètres $x$ et $z$ montre que

$$
\frac{\partial \varphi^{\alpha}}{\partial z^{i}}(x, y, x)=e_{i}^{\alpha}(x, y)
$$

et

$$
\frac{\partial^{2} \varphi^{\alpha}}{\partial z^{i} \partial z^{j}}(x, y, x)=\frac{1}{2}\left(\frac{\partial e_{i}^{\alpha}}{\partial x^{j}}+\frac{\partial e_{j}^{\alpha}}{\partial x^{i}}+\frac{\partial e_{i}^{\alpha}}{\partial y^{\beta}} e_{j}^{\beta}+\frac{\partial e_{j}^{\alpha}}{\partial y^{\beta}} e_{i}^{\beta}\right)(x, y)
$$

en combinant ces deux équations, on obtient

$$
\left(\frac{\partial^{2} \varphi^{\alpha}}{\partial z^{i} \partial x^{j}}+\frac{\partial^{2} \varphi^{\alpha}}{\partial z^{j} \partial x^{i}}+\frac{\partial^{2} \varphi^{\alpha}}{\partial z^{i} \partial y^{\beta}} \frac{\partial \varphi^{\beta}}{\partial z^{j}}+\frac{\partial^{2} \varphi^{\alpha}}{\partial z^{j} \partial y^{\beta}} \frac{\partial \varphi^{\beta}}{\partial z^{i}}\right)(x, y, x)=0 .
$$

Cette remarque permet de préciser le lien entre l'équation (19) et le calcul de Stratonovitch pour des processus continus sur une variété (voir par exemple [4] comme ouvrage de référence en la matière).

\section{2 Énoncé du théorème}

Reformulons dans ce contexte géométrique, le problème de Kurtz, Pardoux et Protter. Partant d'une équation différentielle ordinaire, et d'une semi-martingale discontinue $X$ à valeurs dans une variété, qu'arrive-t-il quand on régularise $X$ en un processus à variation finie continu $X^{h}$, que l'on résout l'équation (17) et que l'on fait tendre $h$ vers $0^{+}$? Le cas où $X$ est à valeurs réelles nous incite à penser que l'on peut considérer la limite comme la solution d'une équation de Stratonovitch avec sauts. 


\section{Serge Cohen et Anne Estrade}

La généralisation de [8] au cas d'un processus directeur à valeurs dans une variété est l'objet du théorème suivant.

THÉORÈME 3.2.- Soit $X$ une semi-martingale à valeurs dans une variété $M$ munie d'un barycentre $\mathbf{B}$ et de son interpolateur associé $I$ et soit $\{e(x, y)\}_{x \in M, y \in N}$ une famille de classe $C^{3}$ en $(x, y)$, à dérivées bornées, d'opérateurs linéaires $e(x, y)$ de $T_{x} M$ dans $T_{y} N$. On considère, pour tout $h>0$, le processus régularisé

$$
X_{t}^{h}(\omega)=\mathbf{B}\left(\mu_{t}^{h} \circ X(\omega)^{-1}\right)
$$

et la solution $Y^{h}$ de l'équation

$$
\mathrm{d} Y^{h}=e\left(X^{h}, Y^{h}\right) \mathrm{d} X^{h}, \quad Y(0)=Y_{0} .
$$

Si

$$
\gamma_{t}=[X, X]_{t}^{d}+t \quad \text { et } \quad \gamma_{t}^{h}=\frac{1}{h} \int_{t-h}^{t} \gamma_{s} \mathrm{~d} s
$$

sont les changements de temps associés respectivement $\grave{a} X$ et $X^{h}$, alors la famille $\left(Y^{h} \circ \gamma_{h}^{-1}\right)_{h>0}$ converge uniformément sur tout compact en probabilité vers un processus adapté continu $\widehat{Y}$ vérifiant $Y=\widehat{Y} \circ \gamma$ où $Y$ est l'unique solution de l'équation

$$
\begin{aligned}
Y^{\alpha}= & y_{0}^{\alpha}+\int_{0} \frac{\partial \varphi^{\alpha}}{\partial z^{i}}\left(X_{s^{-}}, Y_{s^{-}}, X_{s^{-}}\right) \mathrm{d} X_{s}^{i} \\
& +\frac{1}{2} \int_{0} \frac{\partial^{2} \varphi^{\alpha}}{\partial z^{i} \partial z^{j}}\left(X_{s^{-}}, Y_{s^{-}}, X_{s^{-}}\right) \mathrm{d}\left\langle\left(X^{i}\right)^{c},\left(X^{j}\right)^{c}\right\rangle_{s} \\
& +\sum_{0<s \leq .}\left(\varphi^{\alpha}\left(X_{s^{-}}, Y_{s^{-}}, X_{s}\right)-Y_{s^{-}}^{\alpha}-\frac{\partial \varphi^{\alpha}}{\partial z^{i}}\left(X_{s^{-}}, Y_{s^{-}}, X_{s^{-}}\right) \Delta X_{s}^{i}\right) ;
\end{aligned}
$$

avec $\varphi$ construit à partir du flot de (18).

\subsection{Démonstration}

L'idée directrice est de ramener l'équation régularisée (20) à une forme à laquelle on pourra appliquer le théorème $5.4 \mathrm{de}$ [9]. Ce théorème concerne les EDS conduites par des bonnes familles de semi-martingales au sens de la définition 2.11. On prouve de la sorte que la famille des processus définis par $\widehat{Y}^{h}=Y^{h} \circ \gamma_{h}^{-1}$ est relativement compacte pour la topologie de Skorokhod 
sur l'espace des trajectoires càdlàg et on obtient l'équation limite dont est solution toute valeur d'adhérence de la famille $\left(\widehat{Y}^{h}\right)_{h>0}$. Dans la suite, sauf mention spéciale, la relative compacité fera toujours référence à la topologie de Skorokhod sur l'espace des trajectoires càdlàg.

Dégageons ici les trois grandes étapes de la démonstration du théorème 3.2. Dans un premier temps, on opère le changement de temps $\gamma_{h}^{-1}$ sur les processus de l'équation régularisée (20). Après cette transformation, les processus directeurs $\widehat{X}_{h}=X^{h} \circ \gamma_{h}^{-1}$ ne constituant pas une bonne famille, on effectue une intégration par parties qui fait apparaître la bonne famille des processus $A^{h}$. À la fin de cette étape nous aboutissons à l'équation (22) à laquelle nous souhaitons appliquer le théorème $5.4 \mathrm{de}$ [9].

$\mathrm{Au}$ préalable, il nous faut démontrer la relative compacité du terme intégré de l'intégration par parties. C'est l'objet de la deuxième étape, dont la partie la plus importante est l'étude des processus $\widehat{Y}^{h}$ dans un voisinage temporel des instants de discontinuité de $X$; on est en effet amené à comparer la limite des $\widehat{Y}^{h}$ et le flot de l'équation (18).

Enfin, nous montrons la convergence des $\widehat{Y}^{h}$ vers l'unique solution d'une équation différentielle stochastique. Pour obtenir cette équation limite, on étudie séparément les termes qui varient en dehors des intervalles de temps fictifs correspondant aux instants de saut de $X$ (on peut alors utiliser le lemme 2.14 qui fournit la limite des $A^{h}$ ) et les termes qui ne varient qu'aux instants de saut de $X$ (où c'est une équation ordinaire qui régit alors les variations).

Changement de temps et intégration par parties

En effectuant le changement de temps $\gamma_{h}^{-1}$ et en utilisant la remarque 3.1, l'équation (20) devient

$$
\mathrm{d} \widehat{Y}^{h, \alpha}=\frac{\partial \varphi^{\alpha}}{\partial z^{i}}\left(\widehat{X}^{h}, \widehat{Y}^{h}, \widehat{X}^{h}\right) \mathrm{d} \widehat{X}^{h, i}, \quad \hat{Y}^{h, \alpha}(0)=y_{0}^{\alpha}
$$

où $\widehat{X}^{h}=X^{h} \circ \gamma_{h}^{-1}$. Pour obtenir les compacités nécessaires au passage à la limite quand $h$ tend vers 0 , nous introduisons les processus $R^{h, i}=$ $\widehat{X}^{h, i}-X_{\gamma_{h}^{-1}}^{i}$. Le processus $\widehat{Y}^{h}$ vérifie donc le système

$$
\begin{aligned}
\widehat{Y}_{t}^{h, \alpha}= & y_{0}^{\alpha}+\int_{0}^{t} \frac{\partial \varphi^{\alpha}}{\partial z^{i}}\left(\widehat{X}_{s}^{h}, \widehat{Y}_{s}^{h}, \widehat{X}_{s}^{h}\right) \mathrm{d} X_{\gamma_{h}^{-1}(s)}^{i} \\
& +\int_{0}^{t} \frac{\partial \varphi^{\alpha}}{\partial z^{i}}\left(\widehat{X}_{s}^{h}, \widehat{Y}_{s}^{h}, \widehat{X}_{s}^{h}\right) \mathrm{d} R_{s}^{h, i} .
\end{aligned}
$$




\section{Serge Cohen et Anne Estrade}

Or la convergence uniforme de $\gamma_{h}^{-1}$ vers $\gamma^{-1}$ permet d'identifier la limite du premier terme comme une intégrale stochastique où l'intégrant devient $\mathrm{d} X_{\gamma^{-1}}^{i}$. En revanche la famille $\left(R^{h}\right)_{h}$ n'étant pas bonne, on ne peut conclure directement sur le second terme. Nous opérons alors une intégration par parties qui fait apparaître l'intégrant $A^{h(i, j)}$ :

$$
\begin{aligned}
& \int_{0}^{t} \frac{\partial \varphi^{\alpha}}{\partial z^{i}}\left(\widehat{X}_{s}^{h}, \widehat{Y}_{s}^{h}, \widehat{X}_{s}^{h}\right) \mathrm{d} R_{s}^{h, i}= \\
&=\frac{\partial \varphi^{\alpha}}{\partial z^{i}}\left(\hat{X}_{t}^{h}, \widehat{Y}_{t}^{h}, \widehat{X}_{t}^{h}\right) R_{t}^{h, i}+ \\
& \quad-\int_{0}^{t}\left(\frac{\partial^{2} \varphi^{\alpha}}{\partial x^{j} \partial z^{i}}+\frac{\partial^{2} \varphi^{\alpha}}{\partial z^{j} \partial z^{i}}\right)\left(\widehat{X}_{s}^{h}, \widehat{Y}_{s}^{h}, \hat{X}_{s}^{h}\right) R_{s}^{h, i} \mathrm{~d} \widehat{X}_{s}^{h, j}+ \\
&-\int_{0}^{t} \frac{\partial^{2} \varphi^{\alpha}}{\partial y^{\beta} \partial z^{i}}\left(\widehat{X}_{s}^{h}, Y_{s}^{h}, \widehat{X}_{s}^{h}\right) R_{s}^{h, i} \mathrm{~d} \widehat{Y}_{s}^{h, \beta} .
\end{aligned}
$$

D'après l'équation (21) et la défir, ion de $A^{h(i, j)}(\mathrm{cf}(12))$

$$
\begin{aligned}
\int_{0}^{t} \frac{\partial \varphi^{\alpha}}{\partial z^{i}}\left(\widehat{X}_{s}^{h}, \widehat{Y}_{s}^{h}, \widehat{X}_{s}^{h}\right) \mathrm{d} R_{s}^{h, i}= \\
=\frac{\partial \varphi^{\alpha}}{\partial z^{i}}\left(\widehat{X}_{t}^{h}, \widehat{Y}_{t}^{h}, \widehat{X}_{t}^{h}\right) R_{t}^{h, i}+ \\
\quad-\int_{0}^{t}\left(\frac{\partial^{2} \varphi^{\alpha}}{\partial x^{j} \partial z^{i}}+\frac{\partial^{2} \varphi^{\alpha}}{\partial z^{j} \partial z^{i}}+\frac{\partial^{2} \varphi^{\alpha}}{\partial y^{\beta} \partial z^{i}} \frac{\partial \varphi^{\beta}}{\partial z^{j}}\right)\left(\widehat{X}_{s}^{h}, \widehat{Y}_{s}^{h}, \widehat{X}_{s}^{h}\right) \mathrm{d} A_{s}^{h(i, j)},
\end{aligned}
$$

et ainsi le processus $\widehat{Y}^{h}$ est solution du système

$$
\begin{aligned}
\widehat{Y}_{t}^{h, \alpha}= & y_{0}^{\alpha}+U_{t}^{h, \alpha}+\int_{0}^{t} \frac{\partial \varphi^{\alpha}}{\partial z^{i}}\left(\widehat{X}_{s}^{h}, \widehat{Y}_{s}^{h}, \widehat{X}_{s}^{h}\right) \mathrm{d} X_{\gamma_{h}^{-1}(s)}^{i}+ \\
& -\int_{0}^{t}\left(\frac{\partial^{2} \varphi^{\alpha}}{\partial x^{j} \partial z^{i}}+\frac{\partial^{2} \varphi^{\alpha}}{\partial z^{j} \partial z^{i}}+\frac{\partial^{2} \varphi^{\alpha}}{\partial y^{\beta} \partial z^{i}} \frac{\partial \varphi^{\beta}}{\partial z^{j}}\right)\left(\widehat{X}_{s}^{h}, \widehat{Y}_{s}^{h}, \widehat{X}_{s}^{h}\right) \mathrm{d} A_{s}^{h(i, j)}
\end{aligned}
$$

où

$$
U_{t}^{h, \alpha}=\frac{\partial \varphi^{\alpha}}{\partial z^{i}}\left(\widehat{X}_{t}^{h}, \widehat{Y}_{t}^{h}, \widehat{X}_{t}^{h}\right) R_{t}^{h, i}
$$

Pour appliquer le théorème 5.4 de [9], il nous reste encore à établir la relative compacité de la famille $\left(U^{h}\right)_{h}$. 


\section{Compacité de $\left(U^{h}\right)_{h>0}$}

On suppose les trajectoires de la semi-martingale $X$ presque sûrement contenues dans un compact fixe $K$ de la variété $M$, ce qui ne restreint pas la généralité du résultat (cf. remarque 2.4). Pour établir cette compacité, donnons nous une suite à valeurs dans l'ensemble

$$
\left\{U^{h, \alpha} \mid h>0\right\}=\left\{\frac{\partial \varphi^{\alpha}}{\partial z^{i}}\left(\widehat{X}^{h}, \widehat{Y}^{h}, \widehat{X}^{h}\right) R^{h, i} \mid h>0\right\}
$$

et montrons qu'il est possible d'en extraire une sous-suite convergente.

Rappelons que les instants de sauts de $X$ sont notés $\left(\tau_{\ell}\right)_{\ell \geq 1}$. Comme le coefficient $\varphi$ ainsi que ses dérivées partielles sont bornés sur $K$, que les semi-martingales $X \circ \gamma_{h}^{-1}, A^{h(i, j)}$ sont bonnes, et que les $R^{h, i}$ convergent uniformément, la famille $\left(\sup _{t \leq T} \widehat{Y}_{t}^{h}\right)_{h}$ est bornée en probabilité. Ainsi de toute suite $\left(h_{n}\right)_{n}$ tendant vers 0 , on peut extraire une sous-suite encore notée $\left(h_{n}\right)_{n \in \mathbb{N}}$ telle que $\left(\widehat{Y}^{h_{n}} \circ \gamma_{h_{n}}\left(\tau_{\ell}\right)\right)_{\ell \geq 1}$ converge en loi. On note $\left(Y_{\ell}\right)_{\ell \geq 1}$ cette limite et $\Gamma_{h_{n}}$ l'approximation de l'ensemble $\Gamma$ définie par

$$
\Gamma_{h_{n}}=\bigcup_{\ell \geq 1}\left[\gamma_{h_{n}}\left(\tau_{\ell}\right), \gamma_{h_{n}}\left(\tau_{\ell}+h_{n}\right)[.\right.
$$

Compte-tenu de la définition de $R^{h, i}$ et de la convergence de $\gamma_{h}$, il est clair que

$$
\lim _{n \rightarrow \infty} \sup _{t \in[0, T] \backslash \Gamma_{h_{n}}}\left|R_{t}^{h_{n}, i}\right|=0
$$

et donc

$$
\lim _{n \rightarrow \infty} \sup _{t \in[0, T] \backslash \Gamma_{h_{n}}}\left|U_{t}^{h_{n}, \alpha}\right|=0
$$

Reportons à la fin de la preuve de la compacité l'étude de la limite

$$
\begin{gathered}
\lim _{n \rightarrow \infty} \sup _{\gamma_{h_{n}}\left(\tau_{\ell}\right) \leq s<\gamma_{h_{n}}\left(\tau_{\ell}+h_{n}\right)} \mid \widehat{Y}_{s}^{h_{n}, \alpha}+ \\
-\varphi^{\alpha}\left(\frac{s-\gamma_{h_{n}}\left(\tau_{\ell}\right)}{d_{M}\left(X_{\tau_{\ell}}, X_{\tau_{\ell}}\right)^{2}}, X_{\tau_{\ell}^{-}}, \widehat{Y}_{\gamma_{h_{n}}\left(\tau_{\ell}\right)}^{h_{n}}, X_{\tau_{\ell}}\right) \mid=0 \\
-259-
\end{gathered}
$$


Comme $\gamma_{h}\left(\tau_{\ell}\right)$ tend vers $\gamma\left(\tau_{\ell}^{-}\right)=\eta_{1}\left(\gamma\left(\tau_{\ell}\right)\right)$ et $\gamma_{h}\left(\tau_{\ell}+h\right) \rightarrow \gamma\left(\tau_{\ell}\right)=$ $\eta_{2}\left(\gamma\left(\tau_{\ell}\right)\right)$, on déduit de (23) et (24) que les sous-suites $U^{h_{n}, \alpha}$ convergent vers

$$
\begin{aligned}
U_{t}^{\alpha}= & \sum_{\ell} 1_{\left[\gamma\left(\tau_{\ell}^{-}\right), \gamma\left(\tau_{\ell}\right)\right]}(t) \times \\
& \times \frac{\partial \varphi^{\alpha}}{\partial z^{i}}\left(\hat{X}_{t}, \varphi\left(\frac{t-\gamma\left(\tau_{\ell}^{-}\right)}{d_{M}\left(X_{\tau_{\ell}^{-}}, X_{\tau_{\ell}}\right)^{2}}, X_{\tau_{\ell}^{-}}, Y_{\ell}, X_{\tau_{\ell}}\right), \widehat{X}_{t}\right) R_{t}^{i}
\end{aligned}
$$

où $R_{t}^{i}$ désigne la limite (uniforme en $t$ ) de $R_{t}^{h, i}$. Pour finir la preuve de la compacité, il nous reste à montrer (24) qui décrit le comportement à la limite de $\widehat{Y}^{h_{n}} \circ \gamma_{h_{n}}$ au voisinage d'un instant de saut $\tau_{\ell}$ de $X$.

Étude de $\widehat{Y}$ au voisinage des instants de sauts de $X$

Pour simplifier les formules, nous remplaçons $\tau_{\ell}$ par $\tau$ et $h_{n}$ par $h$ quand cela ne porte pas à confusion. De plus, nous posons

$$
\varphi^{h, \alpha}(s)= \begin{cases}\varphi^{\alpha}\left(\frac{s-\gamma_{h}(\tau)}{d_{M}\left(X_{\tau^{-}}, X_{\tau}\right)^{2}}, X_{\tau^{-}}, \hat{Y}^{h} \circ \gamma_{h}(\tau), X_{\tau}\right) \\ \text { si } \frac{s-\gamma_{h}(\tau)}{d_{M}\left(X_{\tau^{-}}, X_{\tau}\right)^{2}} \in[0,1] \\ \hat{Y}^{h} \circ \gamma_{h}(\tau) & \text { si } s-\gamma_{h}(\tau)<0 . \\ \varphi^{\alpha}\left(1, X_{\tau^{-}}, \hat{Y}^{h} \circ \gamma_{h}(\tau), X_{\tau}\right) & \text { si } s-\gamma_{h}(\tau)>d_{M}\left(X_{\tau^{-}}, X_{\tau}\right)^{2} .\end{cases}
$$

Ainsi, la définition du flot de (18) conduit, quand

$$
\frac{s-\gamma_{h}(\tau)}{d_{M}\left(X_{\tau^{-}}, X_{\tau}\right)^{2}} \in[0,1]
$$

à

$$
\begin{aligned}
\varphi^{h, \alpha}(s) & =\widehat{Y}^{h, \alpha} \circ \gamma_{h}(\tau)+ \\
& +\int_{0}^{\frac{s-\gamma_{h}(\tau)}{d_{M}\left(X_{\tau^{-}}, X_{\tau}\right)^{2}}} e_{i}^{\alpha}\left(I\left(u, X_{\tau^{-}}, X_{\tau}\right), \varphi^{h, \alpha}(u)\right) \frac{\mathrm{d}}{\mathrm{d} u} I^{i}\left(u, X_{\tau^{-}}, X_{\tau}\right) \mathrm{d} u .
\end{aligned}
$$

D'autre part, l'équation (21) vérifiée par $\widehat{Y}^{h}$ permet d'écrire

$$
\begin{aligned}
\widehat{Y}_{s}^{h, \alpha}=\widehat{Y}^{h, \alpha} \circ \gamma_{h}(\tau) & +\int_{\gamma_{h}(\tau)}^{s} e_{i}^{\alpha}\left(\widehat{X}_{u}^{h}, \widehat{Y}_{u}^{h}\right) \mathrm{d} \widehat{X}_{u}^{h, i} . \\
& -260-
\end{aligned}
$$


Régularisation d'équations de Stratonovitch à sauts entre variétés

On ramène l'expression de cette équation différentielle de l'intervalle $\left[\gamma_{h}(\tau), s\right]$ à

$$
\left[0, \frac{s-\gamma_{h}(\tau)}{d_{M}\left(X_{\tau^{-}}, X_{\tau}\right)^{2}}\right]
$$

en effectuant le changement de variable

$$
v=\frac{u-\gamma_{h(\tau)}}{d_{M}\left(X_{\tau^{-}}, X_{\tau}\right)^{2}} .
$$

Pour alléger les notations, posons

$$
\tilde{X}_{v}^{h}=\widehat{X}^{h}\left(v d_{M}\left(X_{\tau^{-}}, X_{\tau}\right)^{2}+\gamma_{h}(\tau)\right)
$$

et

$$
\tilde{Y}_{v}^{h}=\widehat{Y}^{h}\left(v d_{M}\left(X_{\tau^{-}}, X_{\tau}\right)^{2}+\gamma_{h}(\tau)\right)
$$

ce dernier processus est solution de l'équation

$$
\begin{aligned}
& \tilde{Y}^{h, \alpha}\left(\frac{s-\gamma_{h(\tau)}}{d_{M}\left(X_{\tau^{-}}, X_{\tau}\right)^{2}}\right)= \\
& \quad=\widehat{Y}^{h, \alpha} \circ \gamma_{h}(\tau)+\int_{0}^{\frac{s-\gamma_{h}(\tau)}{d_{M}\left(X_{\tau^{-}}, X_{\tau}\right)^{2}}} e_{i}^{\alpha}\left(\tilde{X}_{v}^{h}, \tilde{Y}_{v}^{h}\right) \mathrm{d} \tilde{X}_{v}^{h, i} .
\end{aligned}
$$

Ainsi

$$
\tilde{Y}^{h, \alpha}\left(\frac{s-\gamma_{h}(\tau)}{d_{M}\left(X_{\tau^{-}}, X_{\tau}\right)^{2}}\right)
$$

et

$$
\varphi^{\alpha}\left(\frac{s-\gamma_{h}(\tau)}{d_{M}\left(X_{\tau^{-}}, X_{\tau}\right)^{2}}, X_{\tau^{-}}, \widehat{Y}^{h} \circ \gamma_{h}(\tau), X_{\tau}\right)
$$

apparaissent comme les solutions d'une même équation différentielle, où les processus directeurs sont respectivement $\tilde{X}_{u}^{h}$ et $I\left(u, X_{\tau^{-}}, X_{\tau}\right)$. Or la proposition 2.10 et la convergence de $\gamma_{h}(\tau)$ vers $\gamma\left(\tau^{-}\right)$entraînent que $\tilde{X}_{u}^{h}$ converge uniformément vers $\widehat{X}\left(u d_{M}\left(X_{\tau^{-}}, X_{\tau}\right)^{2}+\gamma(\tau)\right)$. D'après le choix fait pour l'interpolateur (définition 2.6), ceci n'est autre que $I\left(u, X_{\tau^{-}}, X_{\tau}\right.$ ) pour $u$ appartenant à un voisinage de $[0,1]$. Dans ce cadre où les processus 


\section{Serge Cohen et Anne Estrade}

sont à variation finie, pour assurer la convergence des intégrales, il suffit de vérifier que presque sûrement la variation totale de $\widetilde{X}^{h}$ sur

$$
\left[0, \frac{\gamma_{h}(\tau+h)-\gamma_{h}(\tau)}{d_{M}\left(X_{\tau^{-}}, X_{\tau}\right)^{2}}\right]
$$

est bornée. En fait,

$$
\int_{0}^{\frac{\gamma_{h}(\tau+h)-\gamma_{h}(\tau)}{d_{M}\left(X_{\tau^{-}}, X_{\tau}\right)^{2}}}\left|\mathrm{~d} \tilde{X}_{s}^{h, i}\right|=\int_{\tau}^{\tau+h}\left|d X_{s}^{h, i}\right|,
$$

donc d'après (14) et pour $h$ assez petit

$$
\begin{aligned}
\int_{0}^{\frac{\gamma_{h}(\tau+h)-\gamma_{h}(\tau)}{d_{M}\left(X_{\tau^{-}}, X_{\tau}\right)^{2}}}\left|\mathrm{~d} \tilde{X}_{s}^{h}\right| & \leq k \frac{C_{K}}{h} \int_{\tau}^{\tau+h} d_{M}\left(X_{s}, X_{s-h}\right) \mathrm{d} s \\
& \leq 2 k C_{K} d_{M}\left(X_{\tau}, X_{\tau^{-}}\right) .
\end{aligned}
$$

Comme les processus $\tilde{X}^{h}$ convergent uniformément vers $I\left(\cdot, X_{\tau^{-}}, X_{\tau}\right)$ et que leurs variations restent bornées, cela montre (24) et conclut l'étude des processus $\widehat{Y}^{h} \circ \gamma_{h}$ au voisinage des instants $\tau_{\ell}$, ainsi que la preuve de la compacité des $U^{h}$.

\section{Convergence de $\left(\widehat{Y}^{h}\right)_{h}$ et identification de la limite}

On va déduire la relative compacité des $\widehat{Y}^{h}$ de celle des $U^{h}$ et du théorème 5.4 de [9]. En effet, on peut extraire une sous-suite $\left(h_{n_{k}}\right)_{k}$ telle que $\left(U^{h_{n_{k}}}, X \circ \gamma_{h_{n_{k}}}^{-1}, A^{h_{n_{k}}}\right)_{k}$ converge en loi pour la topologie de Skorokhod. La famille des processus directeurs $\left(X \circ \gamma_{h_{n_{k}}}^{-1}, A^{h_{n_{k}}}\right)$ de l'équation (22) étant bonne d'après la remarque 2.9 et le lemme 2.12 , le théorème 5.4 de [9] entraîne que $\left(\widehat{Y}^{h}\right)_{h}$ est relativement compacte pour la topologie de Skorokhod et admet donc une sous-suite convergente en loi. Pour prouver la convergence de $\widehat{Y}^{h}$, on montre que toute valeur d'adhérence de la famille $\left(\widehat{Y}^{h}\right)_{h}$ est solution d'une EDS admettant une unique solution forte. Soit $\left(h_{n}\right)_{n}$ une suite tendant vers 0 telle que $\left(U^{h_{n}}, X \circ \gamma_{h_{n}}^{-1}, A^{h_{n}}\right)$ converge en loi vers $\left(U, X \circ \gamma^{-1}, A\right)$ et $\widehat{Y}^{h_{n}}$ converge en loi vers $\widehat{Y}$. Alors, toujours d'après le théorème $5.4, \widehat{Y}$ est solution de

$$
\begin{aligned}
\widehat{Y}_{t}^{\alpha}= & y_{0}^{\alpha}+U_{t}^{\alpha}+\int_{0}^{t} \frac{\partial \varphi^{\alpha}}{\partial z^{i}}\left(\widehat{X}_{s}, \widehat{Y}_{s}, \widehat{X}_{s}\right) \mathrm{d} X_{\gamma^{-1}(s)}^{i}+ \\
& -\int_{0}^{t}\left(\frac{\partial^{2} \varphi^{\alpha}}{\partial x^{j} \partial z^{i}}+\frac{\partial^{2} \varphi^{\alpha}}{\partial z^{j} \partial z^{i}}+\frac{\partial^{2} \varphi^{\alpha}}{\partial y^{\beta} \partial z^{i}} \frac{\partial \varphi^{\beta}}{\partial z^{j}}\right)\left(\widehat{X}_{s}, \widehat{Y}_{s}, \widehat{X}_{s}\right) \mathrm{d} A_{s}^{(i, j)} .
\end{aligned}
$$


D'après le lemme 2.14,

$$
\begin{aligned}
& \int_{0}^{t}\left(\frac{\partial^{2} \varphi^{\alpha}}{\partial x^{j} \partial z^{i}}\right.\left.+\frac{\partial^{2} \varphi^{\alpha}}{\partial z^{j} \partial z^{i}}+\frac{\partial^{2} \varphi^{\alpha}}{\partial y^{\beta} \partial z^{i}} \frac{\partial \varphi^{\beta}}{\partial z^{j}}\right)\left(\widehat{X}_{s}, \widehat{Y}_{s}, \widehat{X}_{s}\right) 1_{\Gamma^{c}}(s) \mathrm{d} A_{s}^{(i, j)}= \\
&=-\frac{1}{2} \int_{0}^{t}\left(\frac{\partial^{2} \varphi^{\alpha}}{\partial x^{j} \partial z^{i}}+\frac{\partial^{2} \varphi^{\alpha}}{\partial z^{j} \partial z^{i}}+\right. \\
&\left.\quad+\frac{\partial^{2} \varphi^{\alpha}}{\partial y^{\beta} \partial z^{i}} \frac{\partial \varphi^{\beta}}{\partial z^{j}}\right)\left(\widehat{X}_{s}, \widehat{Y}_{s}, \widehat{X}_{s}\right) \mathrm{d}\left\langle\left(X^{i}\right)^{c},\left(X^{j}\right)^{c}\right\rangle_{\gamma^{-1}(s)}
\end{aligned}
$$

En utilisant la remarque 3.1 et la symétrie de $\left\langle\left(X^{i}\right)^{c},\left(X^{j}\right)^{c}\right\rangle$, l'équation précédente se simplifie et s'écrit :

$$
\begin{gathered}
\int_{0}^{t}\left(\frac{\partial^{2} \varphi^{\alpha}}{\partial x^{j} \partial z^{i}}+\frac{\partial^{2} \varphi^{\alpha}}{\partial z^{j} \partial z^{i}}+\frac{\partial^{2} \varphi^{\alpha}}{\partial y^{\beta} \partial z^{i}} \frac{\partial \varphi^{\beta}}{\partial z^{j}}\right)\left(\widehat{X}_{s}, \widehat{Y}_{s}, \widehat{X}_{s}\right) 1_{\Gamma^{c}}(s) \mathrm{d} A_{s}^{\left(i,,^{\prime}\right)}= \\
=-\frac{1}{2} \int_{0}^{t} \frac{\partial^{2} \varphi^{\alpha}}{\partial z^{j} \partial z^{i}}\left(\widehat{X}_{s}, \widehat{Y}_{s}, \widehat{X}_{s}\right) \mathrm{d}\left\langle\left(X^{i}\right)^{c},\left(X^{j}\right)^{c}\right\rangle_{\gamma^{-1}(s)}
\end{gathered}
$$

Finalement $\widehat{Y}$ est solution de :

$$
\begin{aligned}
\widehat{Y}_{t}^{\alpha} & = \\
= & y_{0}^{\alpha}+U_{t}^{\alpha} \\
& -\int_{0}^{t}\left(\frac{\partial^{2} \varphi^{\alpha}}{\partial x^{j} \partial z^{i}}+\frac{\partial^{2} \varphi^{\alpha}}{\partial z^{j} \partial z^{i}}+\frac{\partial^{2} \varphi^{\alpha}}{\partial y^{\beta} \partial z^{i}} \frac{\partial \varphi^{\beta}}{\partial z^{j}}\right)\left(\widehat{X}_{s}, \widehat{Y}_{s}, \widehat{X}_{s}\right) \mathbf{1}_{\Gamma}(s) \mathrm{d} A_{s}^{(i, j)} \\
& +\int_{0}^{t} \frac{\partial \varphi^{\alpha}}{\partial z^{i}}\left(\widehat{X}_{s}, \widehat{Y}_{s}, \widehat{X}_{s}\right) \mathrm{d} X_{\gamma^{-1}(s)}^{i} \\
& +\frac{1}{2} \int_{0}^{t} \frac{\partial^{2} \varphi^{\alpha}}{\partial z^{j} \partial z^{i}}\left(\widehat{X}_{s}, \widehat{Y}_{s}, \widehat{X}_{s}\right) \mathrm{d}\left\langle\left(X^{i}\right)^{c},\left(X^{j}\right)^{c}\right\rangle_{\gamma^{-1}(s)} .
\end{aligned}
$$

Or nous savons que $\widehat{Y}$ suit le flot de l'équation ordinaire (18) sur chaque intervalle $\left[\gamma\left(\tau_{\ell}^{-}\right), \gamma\left(\tau_{\ell}\right)\right]$ d'après $(24)$, et vérifie donc $: \forall t \in\left[\gamma\left(\tau_{\ell}^{-}\right), \gamma\left(\tau_{\ell}\right)\right]$

$$
\hat{Y}_{t}^{\alpha}=\varphi^{\alpha}\left(\frac{t-\gamma\left(\tau_{\ell}^{-}\right)}{d_{M}\left(X_{\tau_{\ell}^{-}}, X_{\tau_{\ell}}\right)^{2}}, X_{\tau_{\ell}^{-}}, \widehat{Y} \circ \gamma\left(\tau_{\ell}^{-}\right), X_{\tau_{\ell}}\right) \text {. }
$$

La suite des équations (31) quand $\ell$ varie détermine $\widehat{Y}$ sur $\Gamma$; de plus, la semi-martingale définie par $(29)+(30)$ est constante sur $\Gamma$ sauf au point de la forme $\gamma\left(\tau_{\ell}^{-}\right)$où elle saute de

$$
\begin{gathered}
\frac{\partial \varphi^{\alpha}}{\partial z^{i}}\left(\widehat{X}_{\gamma\left(\tau_{\ell}^{-}\right)}, \widehat{Y}_{\gamma\left(\tau_{\ell}^{-}\right)}, \widehat{X}_{\gamma\left(\tau_{\ell}^{-}\right)}\right) \Delta X_{\tau_{\ell}}^{i} . \\
-263-
\end{gathered}
$$


On obtient ainsi une équation équivalente à (26) :

$$
\begin{aligned}
\widehat{Y}_{t}^{\alpha}= & y_{0}^{\alpha}+ \\
+ & \sum_{\ell} 1_{\left[\gamma\left(\tau_{\ell}^{-}\right),+\infty[\right.}(t)\left\{\varphi^{\alpha}\left(\frac{t \wedge \gamma\left(\tau_{\ell}\right)-\gamma\left(\tau_{\ell}^{-}\right)}{d_{M}\left(X_{\tau_{\ell}^{-}}, X_{\tau_{\ell}}\right)^{2}}, X_{\tau_{\ell}^{-}}, \widehat{Y} \circ \gamma\left(\tau_{\ell}^{-}\right), X_{\tau_{\ell}}\right)\right. \\
& \left.\quad-\hat{Y}^{\alpha} \circ \gamma\left(\tau_{\ell}^{-}\right)-\frac{\partial \varphi^{\alpha}}{\partial z^{i}}\left(X_{\tau_{\ell}^{-}}, \widehat{Y} \circ \gamma\left(\tau_{\ell}^{-}\right), X_{\tau_{\ell}}\right) \Delta X_{\tau_{\ell}}^{i}\right\} \\
+ & \int_{0}^{t} \frac{\partial \varphi^{\alpha}}{\partial z^{i}}\left(\hat{X}_{s}, \widehat{Y}_{s}, \widehat{X}_{s}\right) \mathrm{d} X_{\gamma^{-1}(s)}^{i} \\
+ & \frac{1}{2} \int_{0}^{t} \frac{\partial^{2} \varphi^{\alpha}}{\partial z^{j} \partial z^{i}}\left(\widehat{X}_{s}, \widehat{Y}_{s}, \widehat{X}_{s}\right) \mathrm{d}\left\langle\left(X^{i}\right)^{c},\left(X^{j}\right)^{c}\right\rangle_{\gamma^{-1}(s)} .
\end{aligned}
$$

Or cette équation localisée admet une unique solution forte et ceci assure la convergence des $\left(\widehat{Y}^{h}\right)_{h}$ vers l'unique solution forte de (32). Donc $\widehat{Y} \circ \gamma$ vérifie l'équation (19).

Enfin pour obtenir la convergence en probabilité de $\widehat{Y}^{h}$ vers $\widehat{Y}$, on remarque que le couple $\left(X \circ \gamma_{h}^{-1}, A^{h}\right)$ converge en probabilité, et on applique le corollaire 5.6 de [9] à l'équation (26).

De plus, comme dans [8], on peut déduire le corollaire suivant.

CoRollaire 3.3. - La suite $\left(Y_{t}^{h}\right)_{h}$ converge en probabilité vers $Y_{t}$ en dehors des instants $t$ de discontinuité du processus directeur $X$.

\section{Rérérences}

[1] AHN (H.) .- Semimartingale representation and the Wong-Zakai problem, $\mathrm{PhD}$ thesis, Purdue University, 1994.

[2] COHEN (S.) .- Géométrie différentielle stochastique avec sauts 1, Stochastics and Stochastic Reports 56 (1996), pp. 179-203.

[3] COHEN (S.) .- Géométrie différentielle stochastique avec sauts 2 : discrétisation et applications des eds avec sauts, Stochastics and Stochastic Reports 56 (1996), pp. 205225.

[4] EMEry (M.) .- Stochastic Calculus on Manifolds, Universitext, Springer, 1989.

[5] EMERY (M.) et MOKOBODZKI (G.) .- Sur le barycentre d'une probabilité dans une variété, Séminaire de Probabilités XXV, Lecture Notes in Mathematics 1485 (1991). 
Régularisation d'équations de Stratonovitch à sauts entre variétés

[6] Jakubowski (A.), MÉmin (J.) et Pagès (G.) .- Convergences en loi des suites d'intégrales stochastiques sur l'espace $D^{1}$ de Skorokhod, Proba. Theory and Related Fields 81 (1989), pp. 111-137.

[7] KURTZ (T. G.) .- Random time changes and convergence in distribution under the Meyer-Zheng conditions, Annals of Probability 19 (1991), pp. 1010-1034.

[8] Kurtz (T. G.), Pardoux (E.). et Protter (P.) .- Stratonovich stochastic differential equations driven by general semimartingales, Annales de l'Institut HenriPoincaré 31, n 2 (1995), pp. 351-378.

[9] KURTz (T. G.) et PROTTER (P.) .- Weak limit theorems for stochastic integrals and stochastic differential equations, Annals of Probability 19 (1991), pp. 1035-1070.

[10] PICARD (J.) .- Barycentres et martingales sur une variété, Annales de l'Institut Henri-Poincaré 30, n 4 (1994), pp. 647-702.

[11] Protter (P.) .- Approximations of solutions of stochastic differential equations driven by semimartingales, Annals of Probabability 13 (1985), pp. 716-743.

[12] Russo (F.) et VALLOIS (P.) .- The generalized quadratic variation process and Itô formula, Stochastic Processes and their Applications, 59 (1995), pp. 81-104.

[13] WONG (E.) et $\mathrm{Z}_{\mathrm{AKAI}}$ (M.) .- On the convergence of ordinary integrals to stochastic integrals, Ann. Math. Statist. 36 (1965), pp. 1560-1564. 